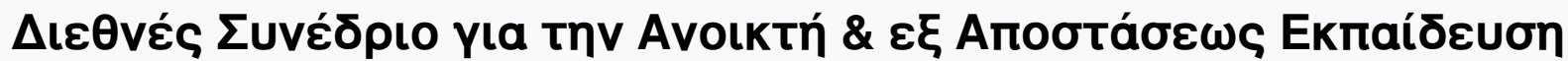

Tón. 7, Ap. 1A (2013)

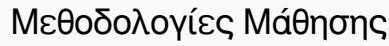

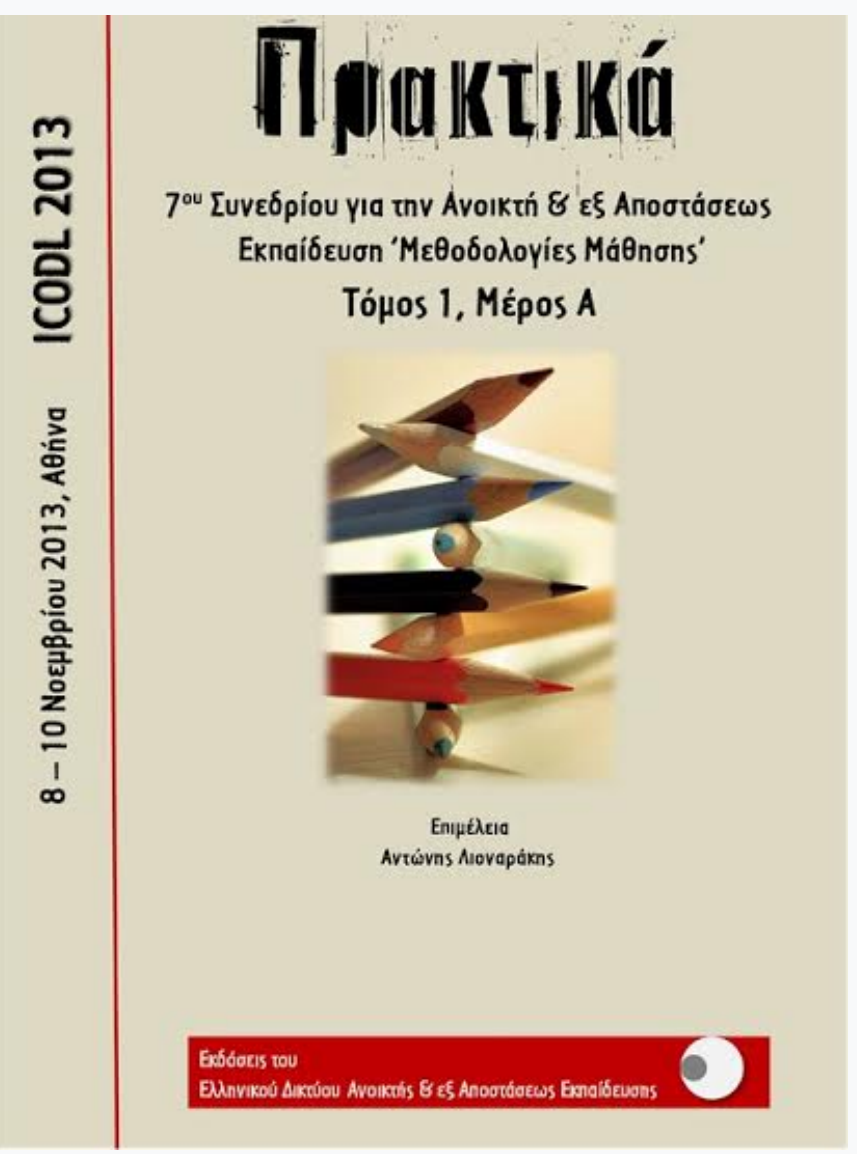

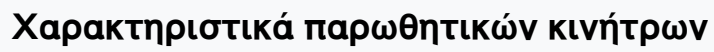

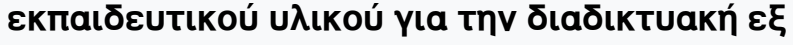

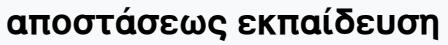

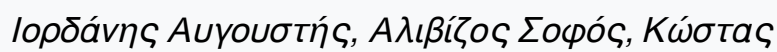
Апо́бтодос

doi: $\underline{10.12681 / \text { icodl. } 541}$ 


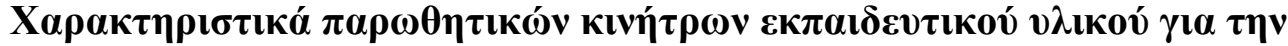

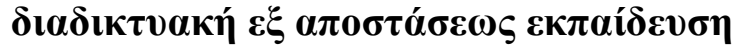

\section{Features of motivational design for e-learning}

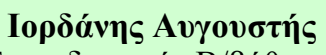

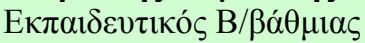 \\ $\varepsilon \kappa \pi \alpha i ́ \delta \varepsilon v \sigma \eta \varsigma$ \\ premnt11002@aegean.gr
}

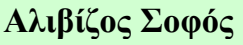

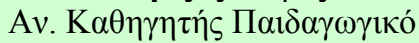

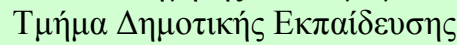

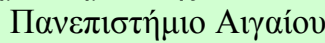 \\ lsofos@aegean.gr
}

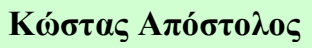

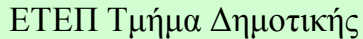

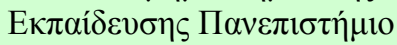 \\ Aryaíov \\ apkostas@aegean.gr
}

\begin{abstract}
One of the main issues that e learning teachers call into question, is the quality of the educational material, since this constitutes the main tool and facilitator of teaching, due to the fact that the teacher's physical appearance is absent. The right formation of the educational material gives fundamental motivation for learning and for this reason the strategies for this formation compose important reason for research. The adaptation of an educational text into a text able to increase motivation for learning is one of the main aims of an educational material designer.

This present research aims at using a tool, which supplies motivation, (model ARCS) accredited by the international bibliography in order to transform, through the use of suitable strategies, a simple teaching text into a dynamic one .

Thus, taking the opportunity of one of the life long learning programmes rendered by the University of the Aegean, with the use of appropriate methodology, a dynamic text was compared to a simple one. Specifically, the participants in the program me were questioned on how much they learned after being taught a module consisting of a dynamic text and if they had continuous motivation for learning. The results were impressive and various. Obviously, the interest of the trainees at the specific workshop proved to be bigger and reinforced.
\end{abstract}

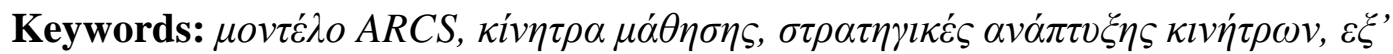
$\alpha \pi o \sigma \tau \alpha \dot{\sigma \varepsilon} \omega \varsigma \varepsilon \kappa \pi \alpha i \delta \varepsilon v \sigma \sigma \eta$, e-learning, model ARCS, motivation

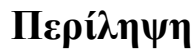

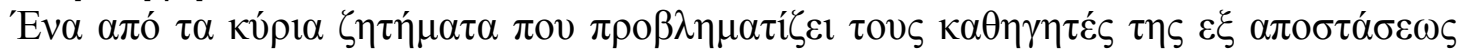

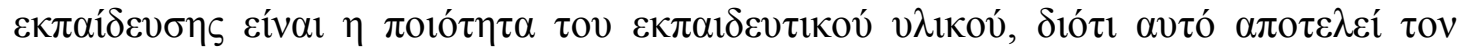

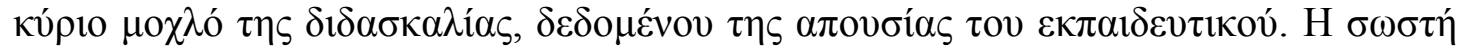

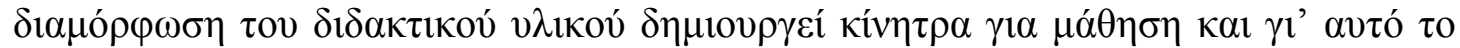

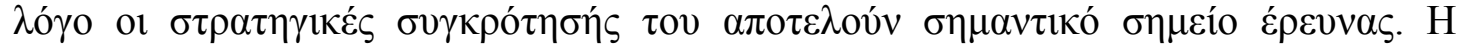

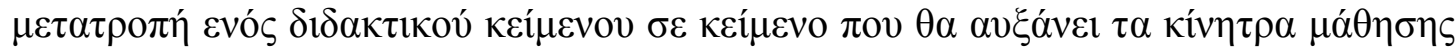

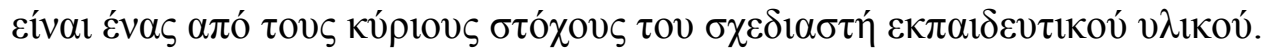

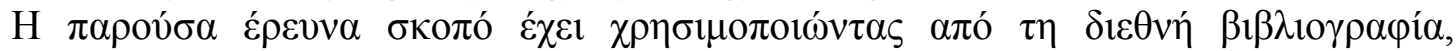

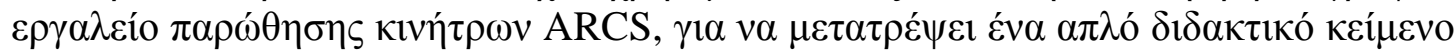

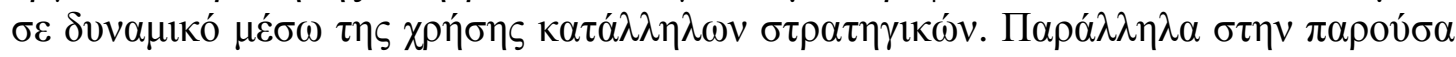

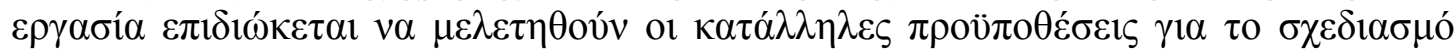

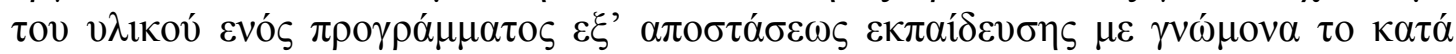




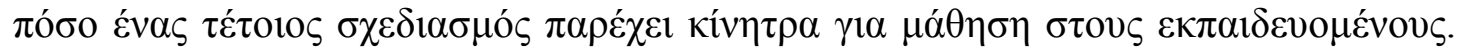

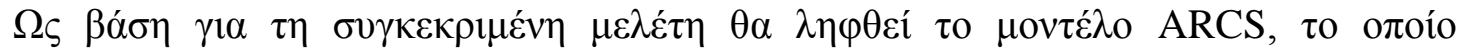

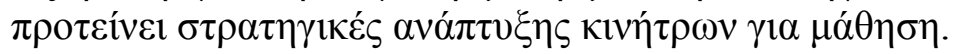

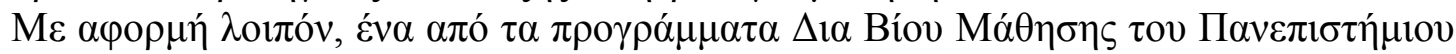

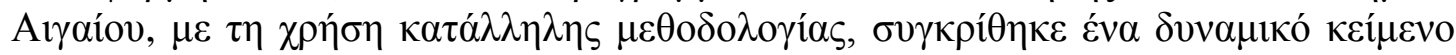

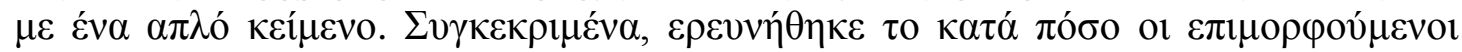

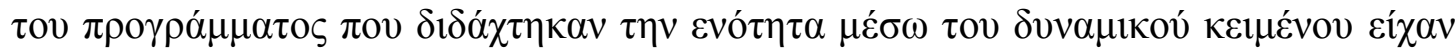

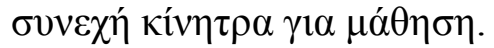

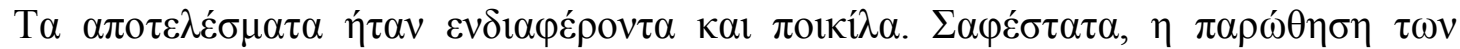

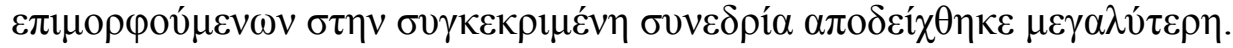

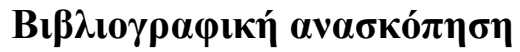

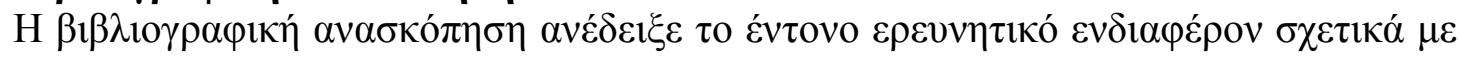

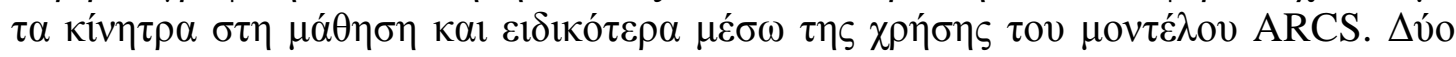

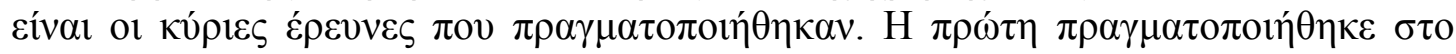

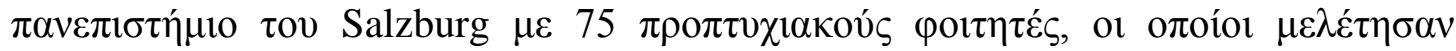

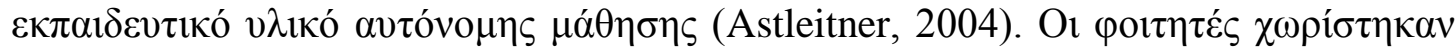

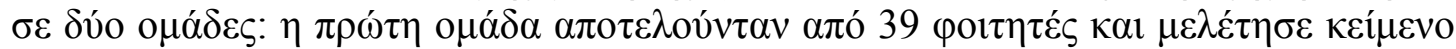

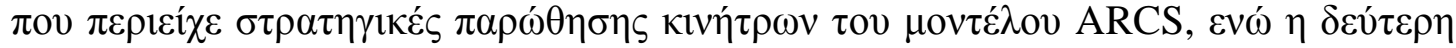

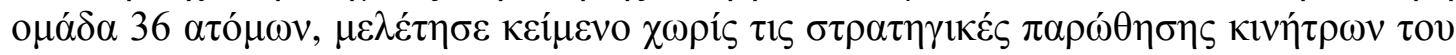

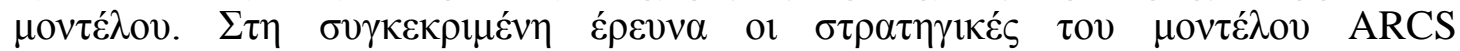

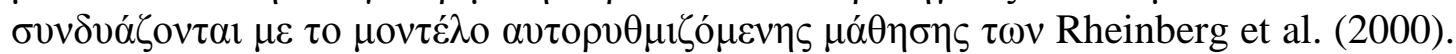

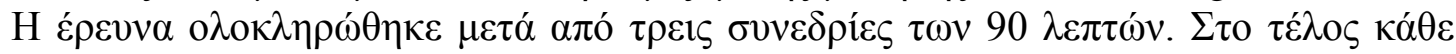

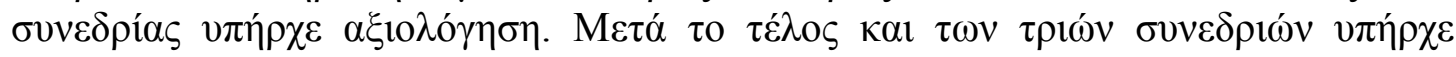

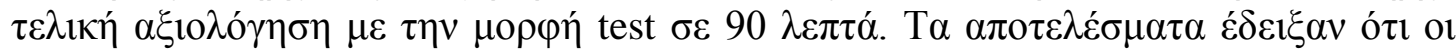

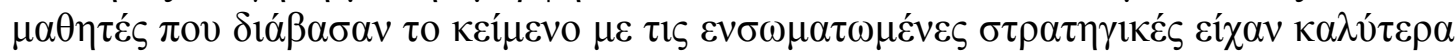

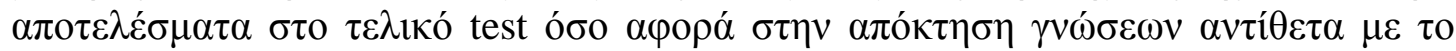

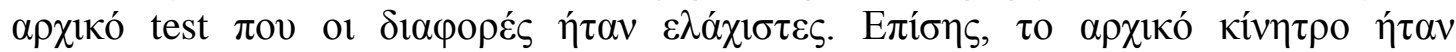

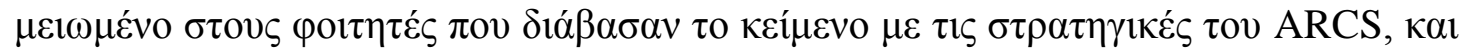

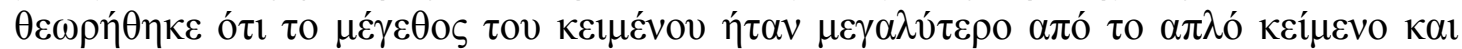

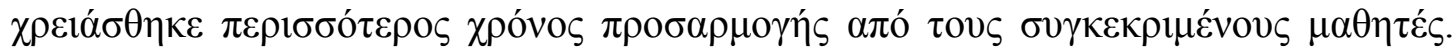

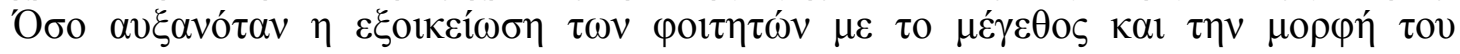

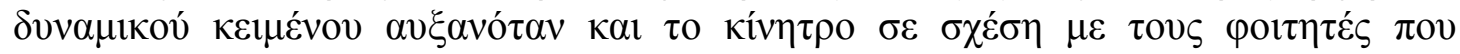

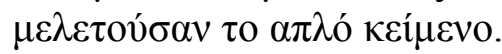

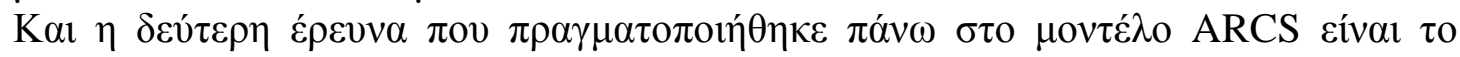

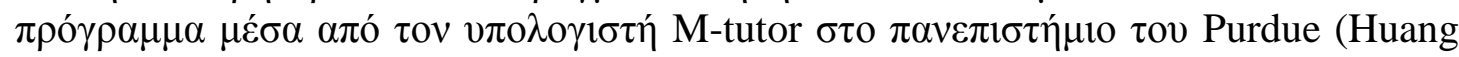

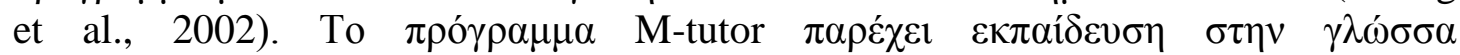

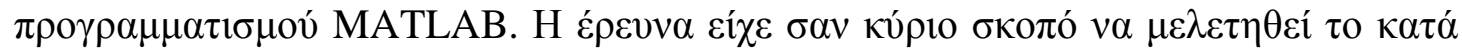

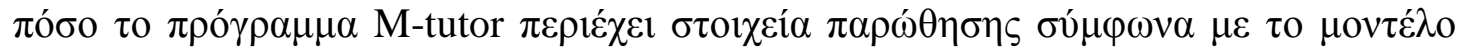

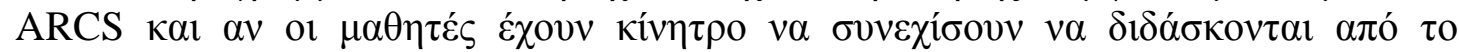

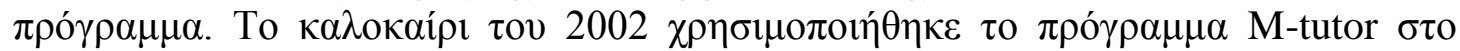

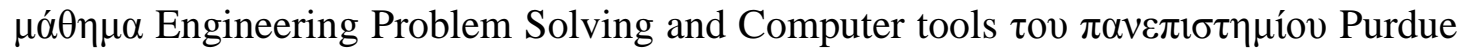

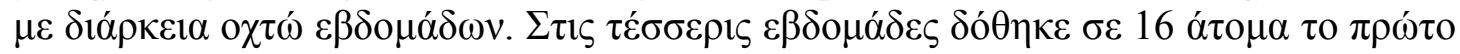

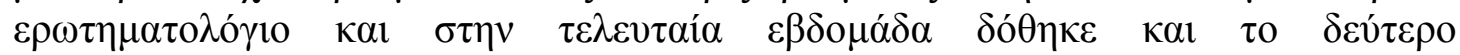

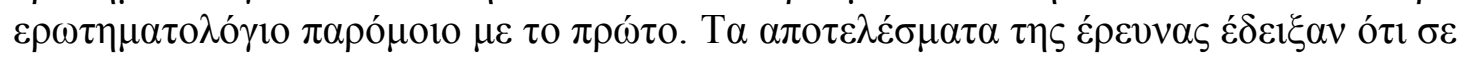

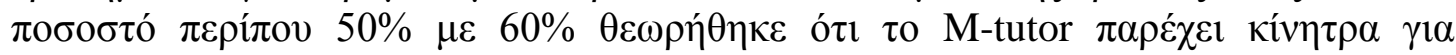

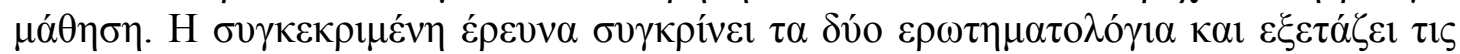

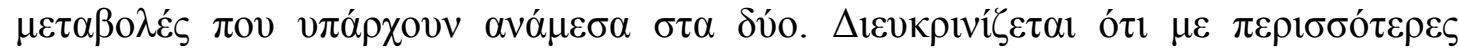

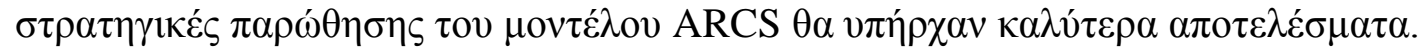




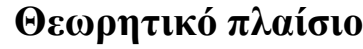

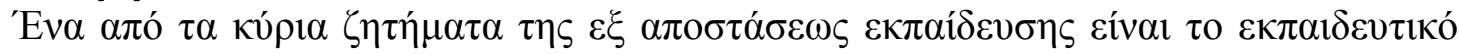

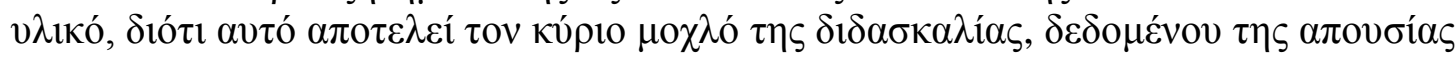

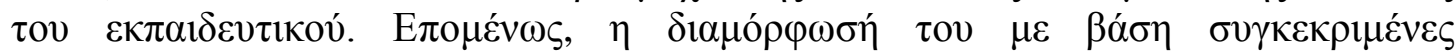

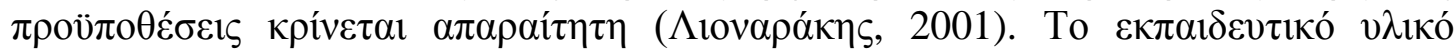

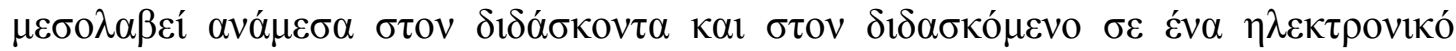

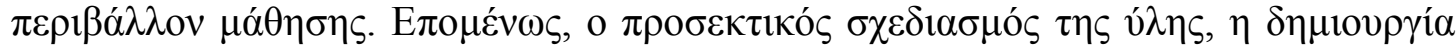

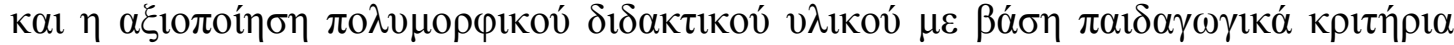

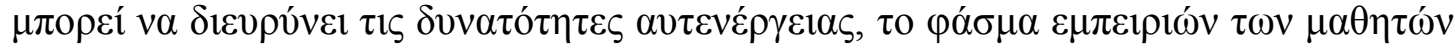

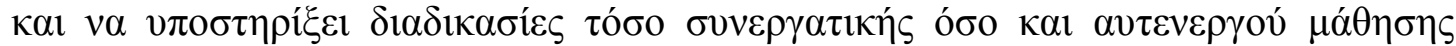

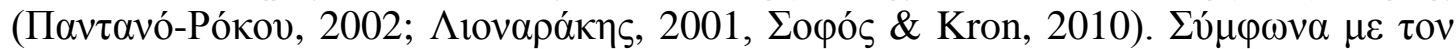

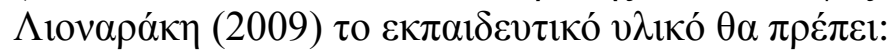

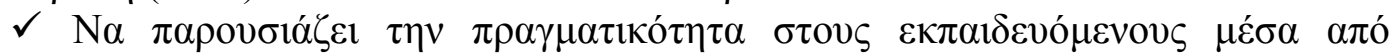

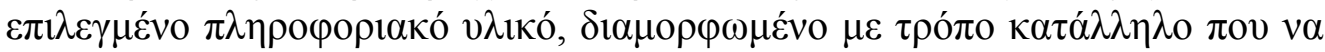

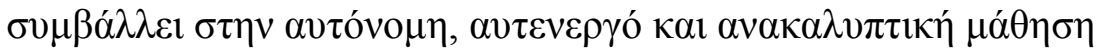

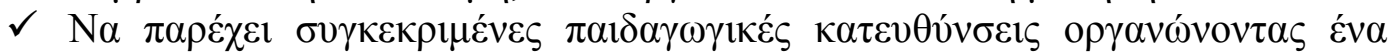

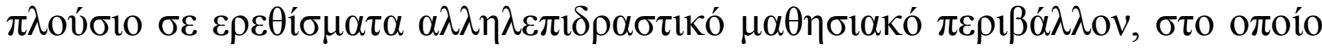

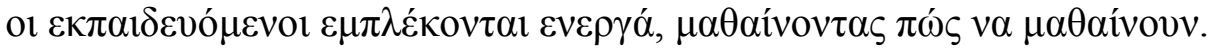

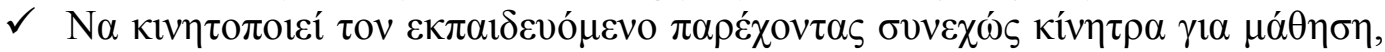

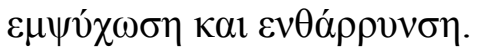

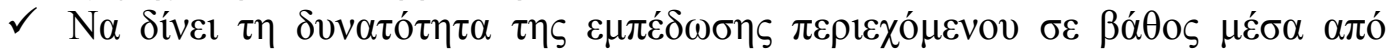

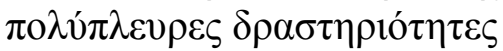

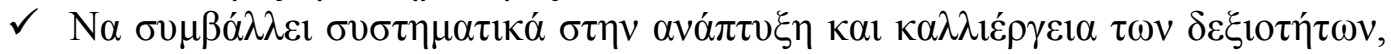

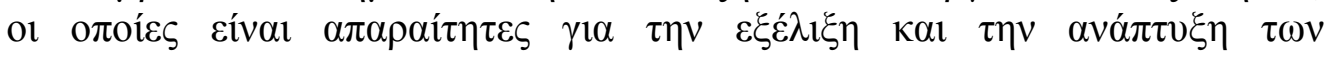
$\varepsilon \kappa \pi \alpha 1 \delta \varepsilon v 0 \mu \varepsilon ́ v \omega v$.

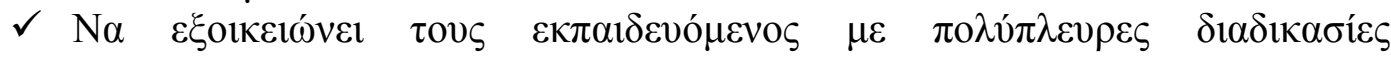
$\alpha \xi 10 \lambda \hat{\gamma} \eta \sigma \eta s$.

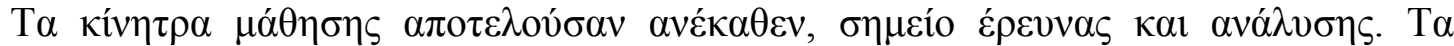

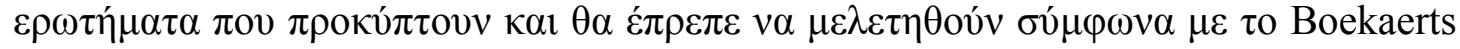

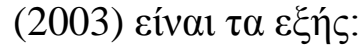

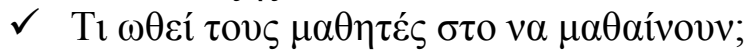

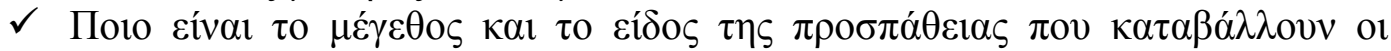
$\mu \alpha \theta \eta \tau \dot{\varepsilon} \varsigma \gamma 1 \alpha \tau \eta \mu \alpha \dot{\theta} \theta \eta \sigma \eta$

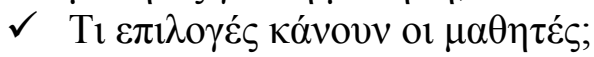

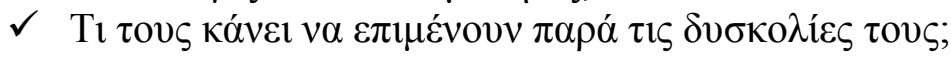

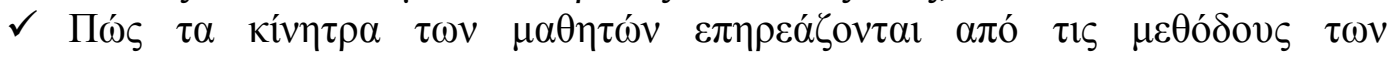

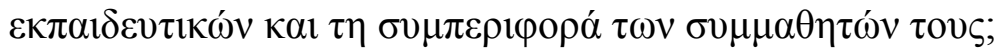

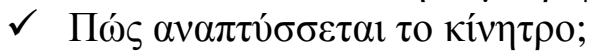

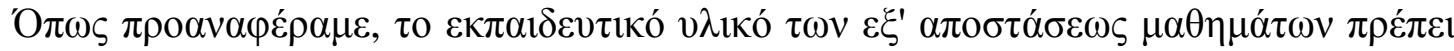

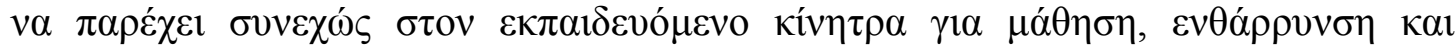

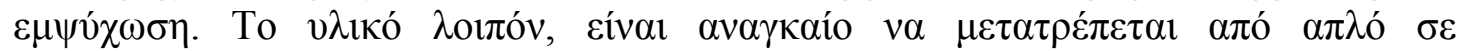

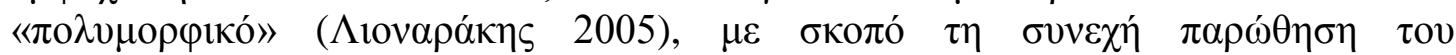

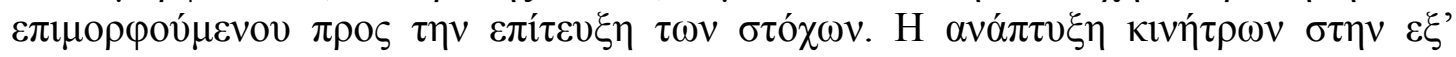

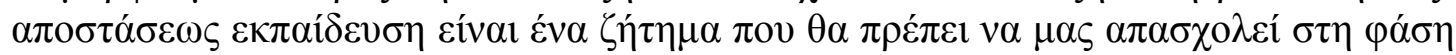

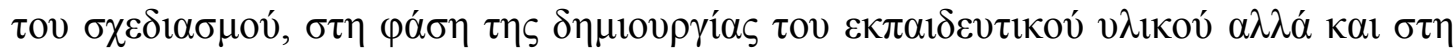

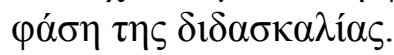

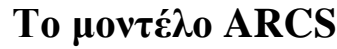




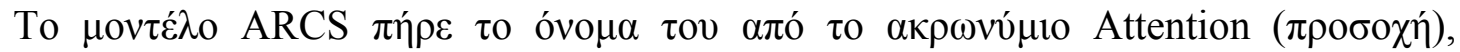

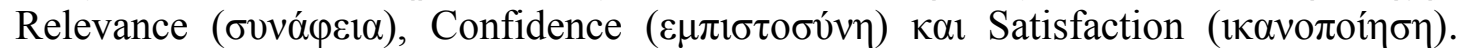

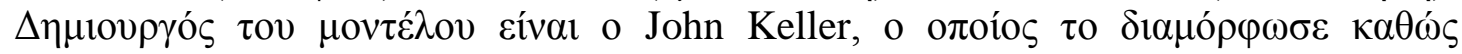

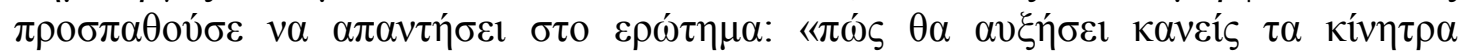

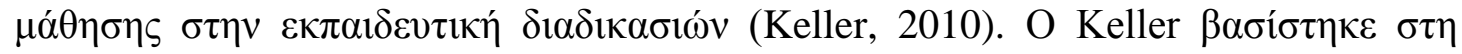

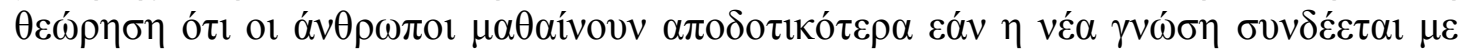

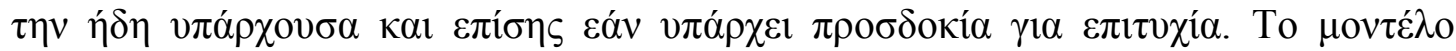

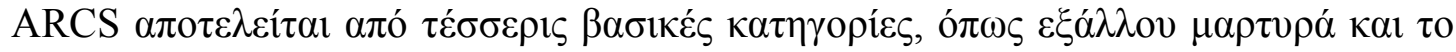

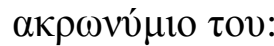

$\checkmark$ Пробохй,

$\checkmark \Sigma v v \alpha ́ \varphi \varepsilon i \alpha$,

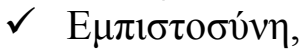

$\checkmark$ Ікаvолоі́

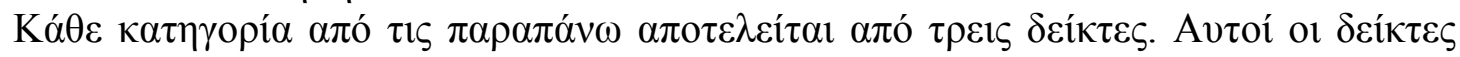

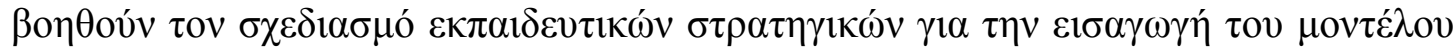

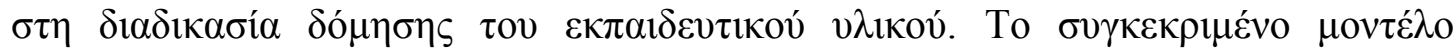

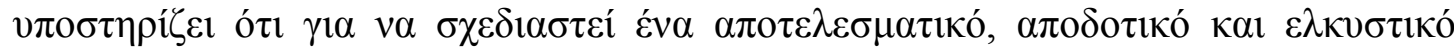
$\varepsilon \kappa \pi \alpha 1 \delta \varepsilon v \tau \imath \kappa o ́ ~ v \lambda ı к o ́ ~ \pi \rho \varepsilon ́ \pi \varepsilon 1 ~ v \alpha ~ \lambda \alpha \mu \beta \alpha ́ v \varepsilon 1 ~ v \pi o ́ \psi \eta ~ \alpha v \tau o u ́ \varsigma ~ \tau o v \varsigma ~ \tau \varepsilon ́ \sigma \sigma \varepsilon \rho 1 \varsigma ~ \pi \alpha \rho \alpha ́ \gamma o v \tau \varepsilon \varsigma$,

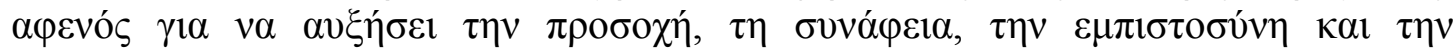

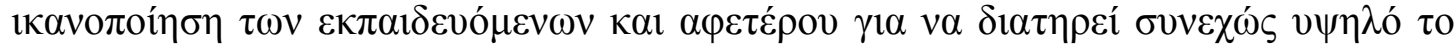

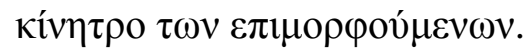

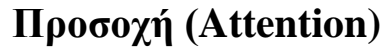

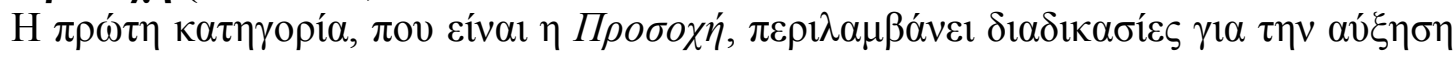

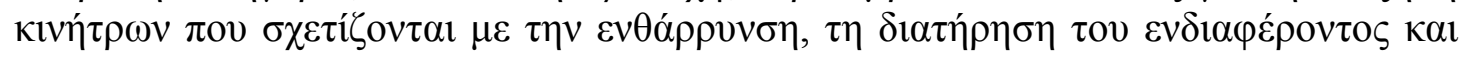

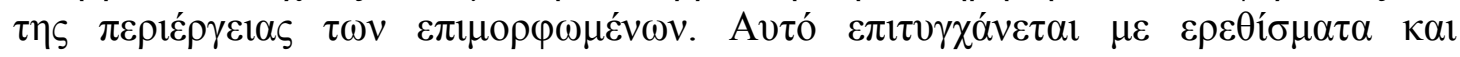

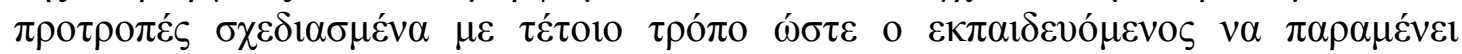

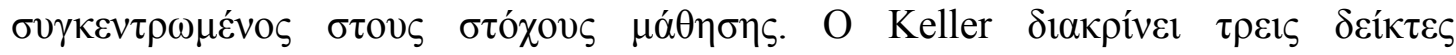
«Пробохиंs»:

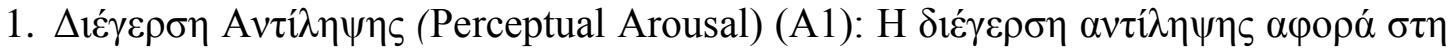

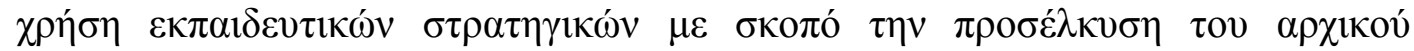

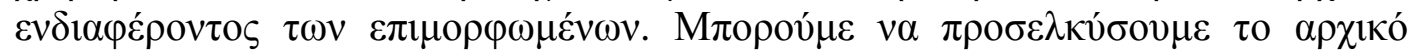

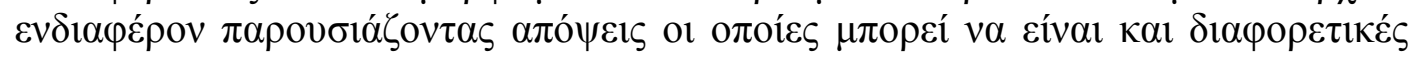

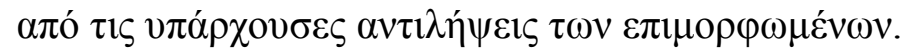

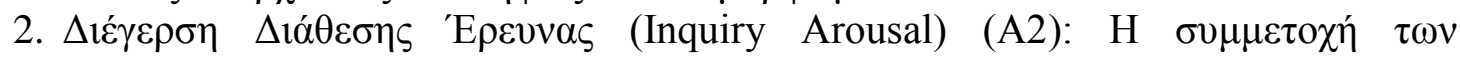

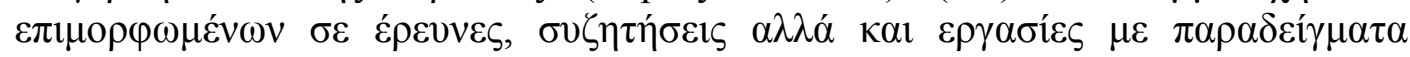

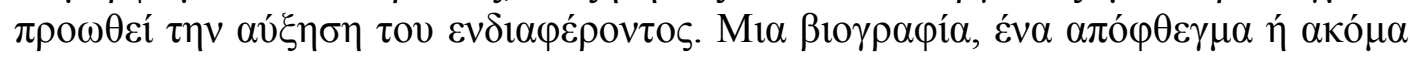

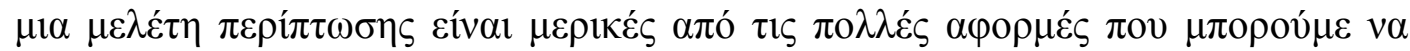

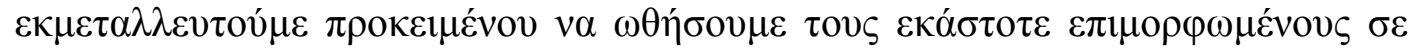
$\pi \varepsilon \rho \alpha \imath \varepsilon \dot{\varepsilon} \rho \omega \mu \lambda \varepsilon \dot{\varepsilon} \tau$.

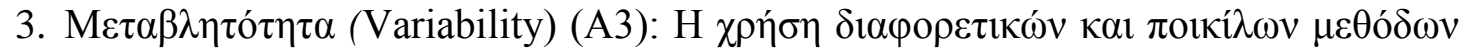

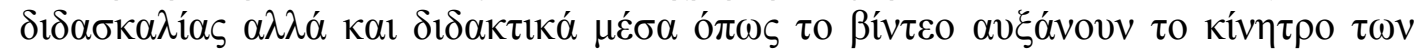

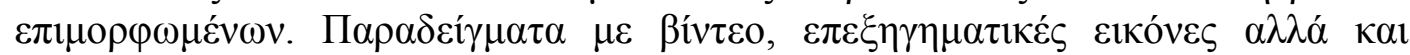

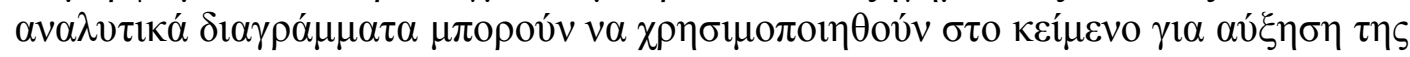

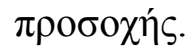

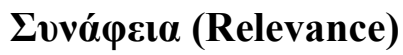

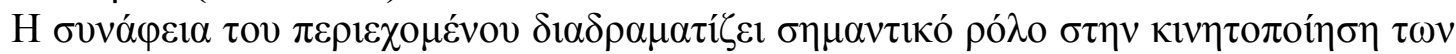

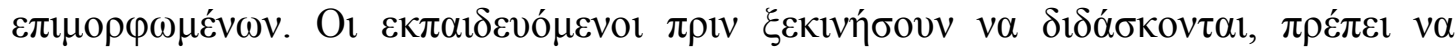

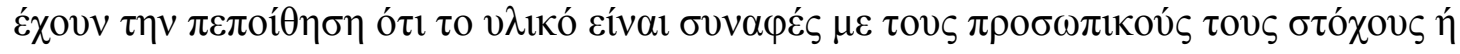




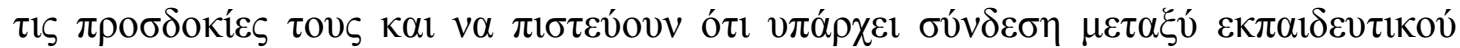

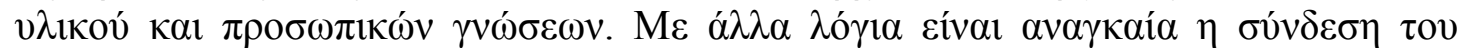

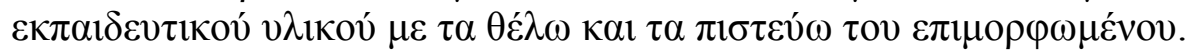

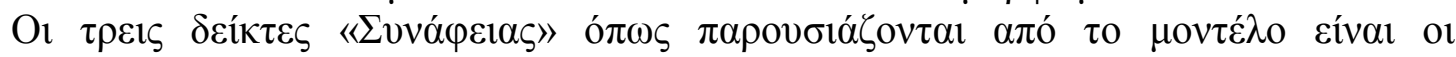
$\pi \alpha \rho \alpha \kappa \alpha ́ \tau \omega$ :

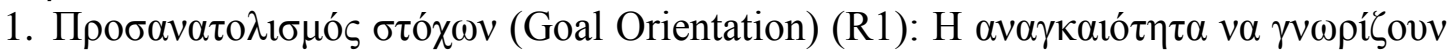

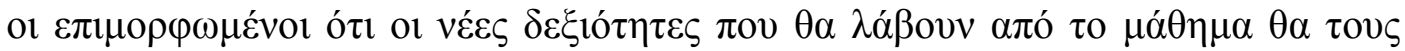

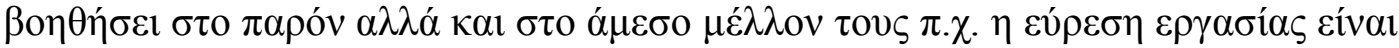

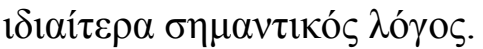

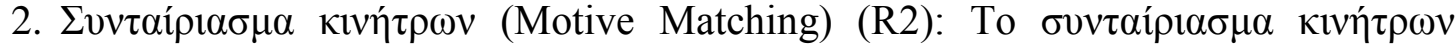

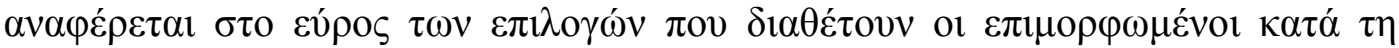

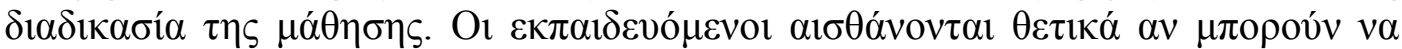

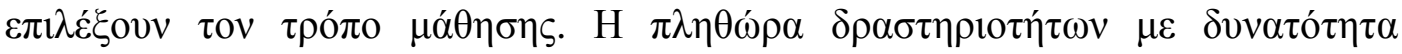

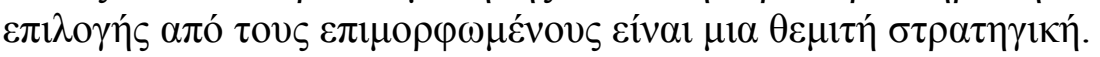

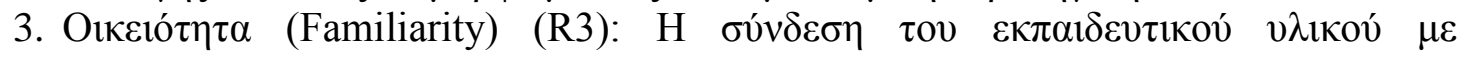

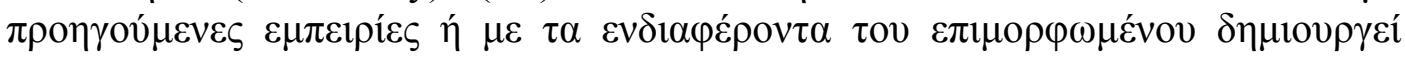

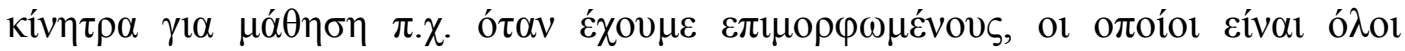

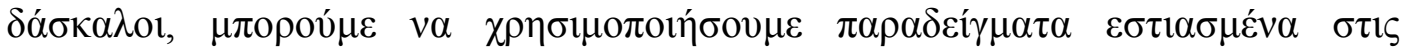

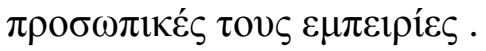

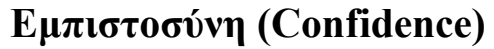

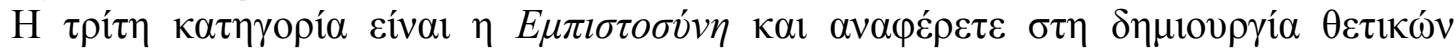

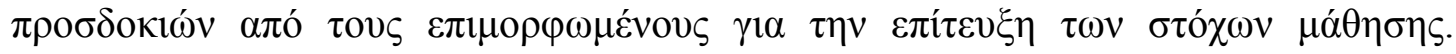

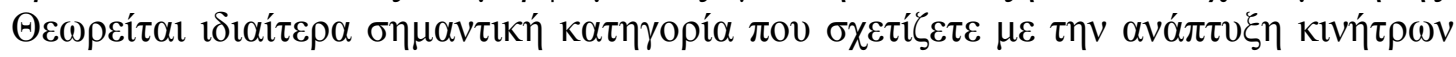

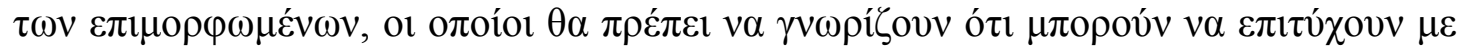

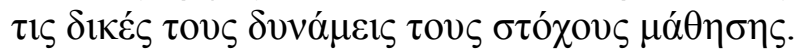

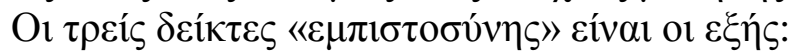

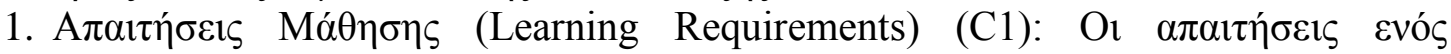

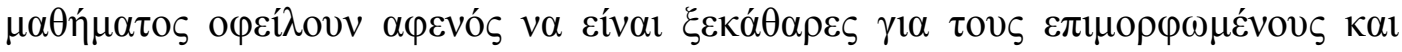

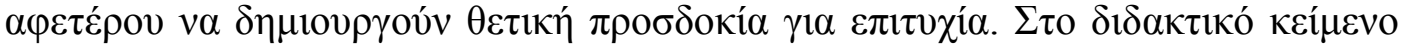

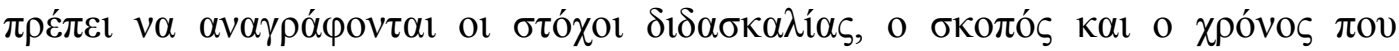

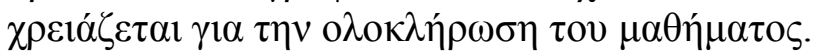

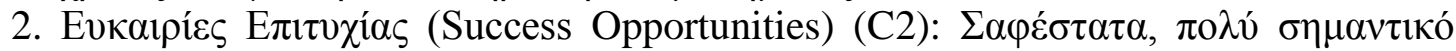

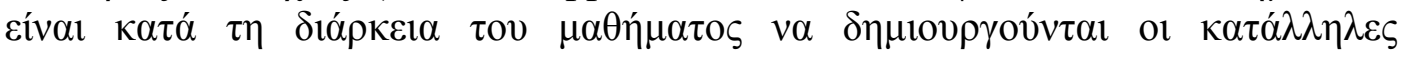

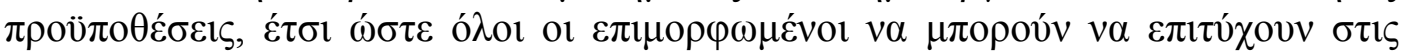

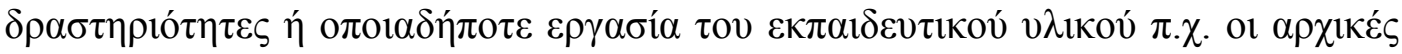

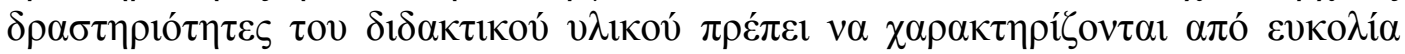

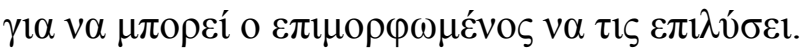

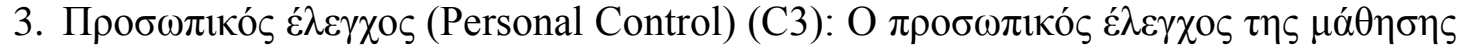

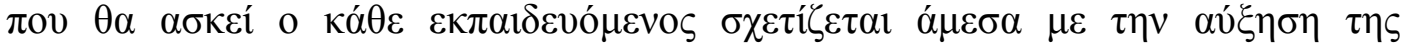

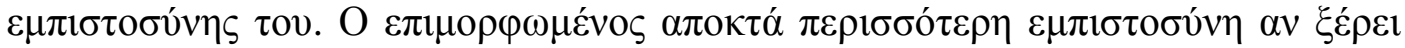

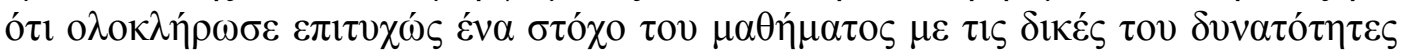

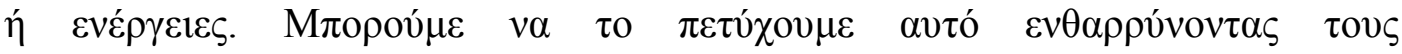

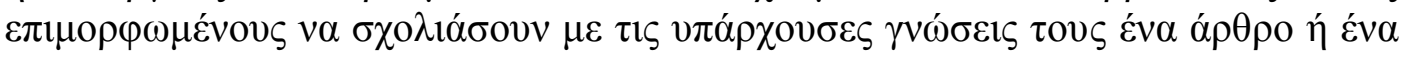
$\pi \alpha \rho \alpha ́ \delta \varepsilon 1 \gamma \mu \alpha$.

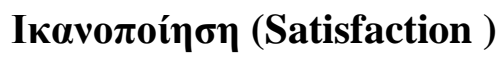

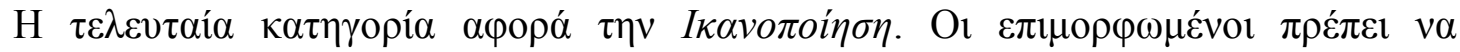

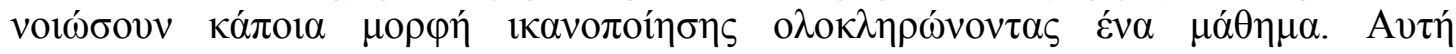

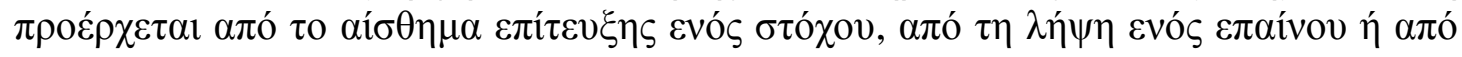




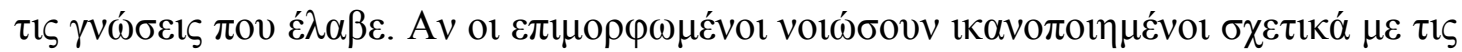

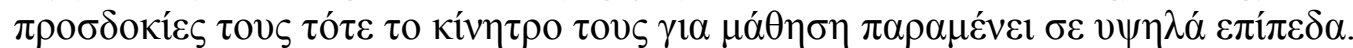

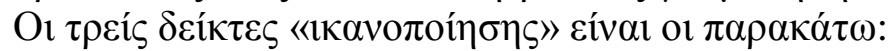

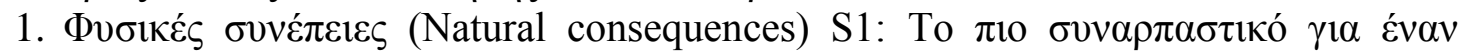

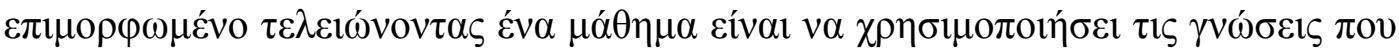

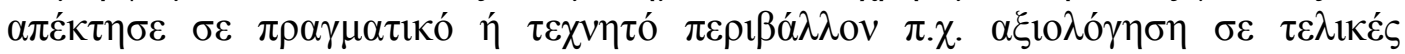
$\varepsilon \rho \gamma \alpha \sigma i ́ \varepsilon \varsigma$.

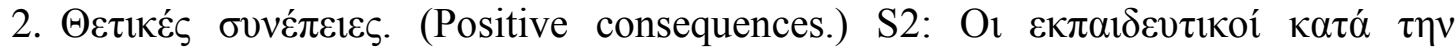

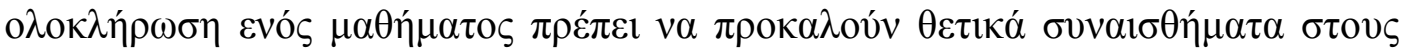

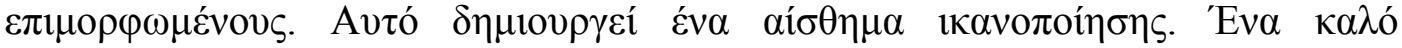

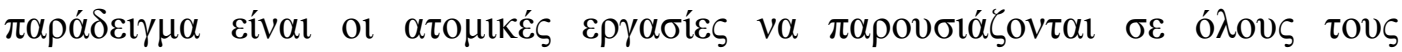

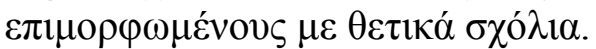

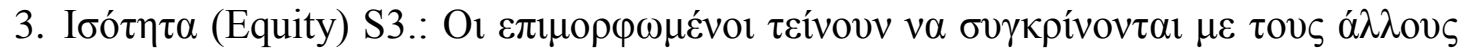

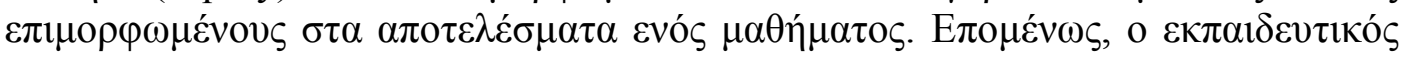

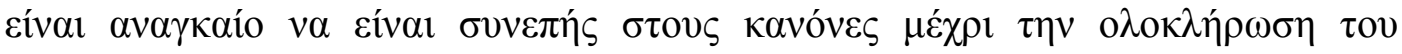

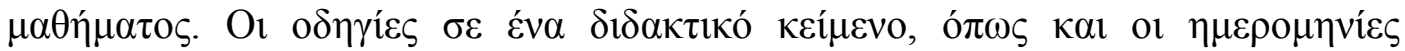

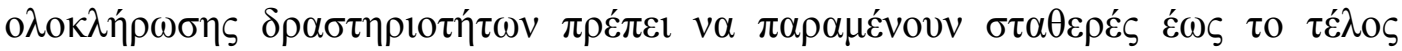

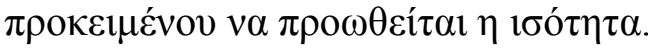

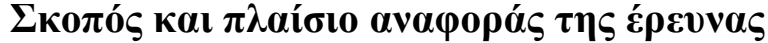

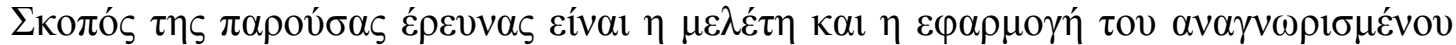

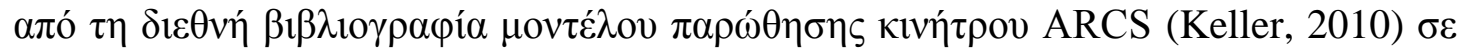

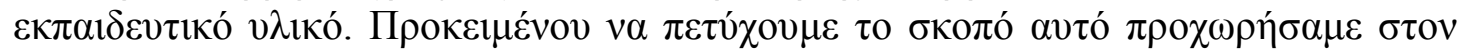

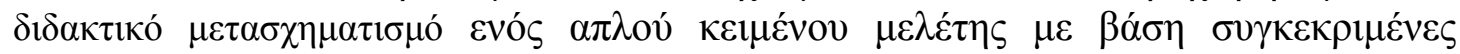

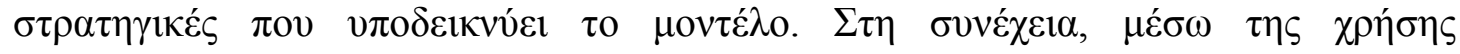

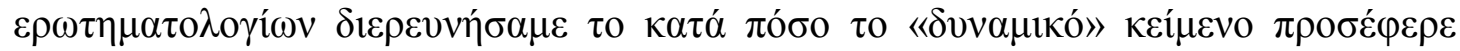

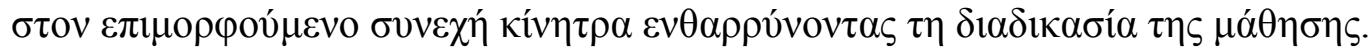

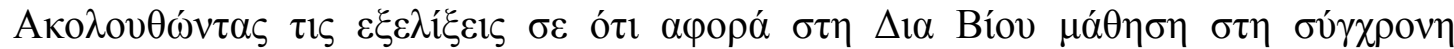

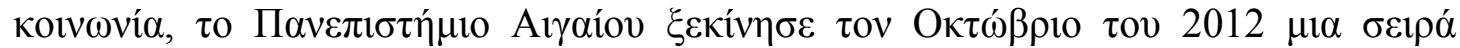

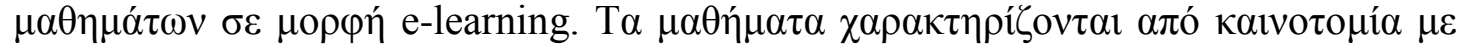

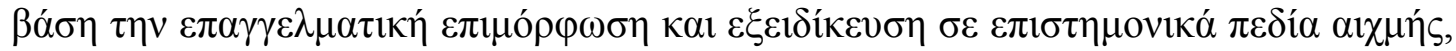

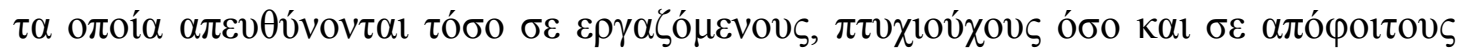

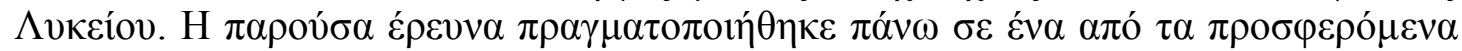

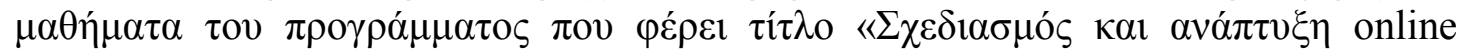
$\mu \alpha \theta \eta \mu \alpha ́ \tau \omega v »$.

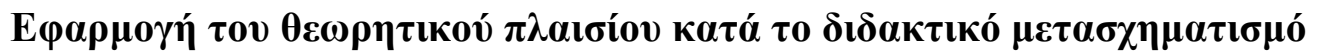

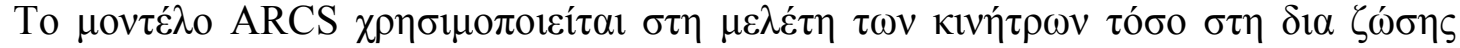

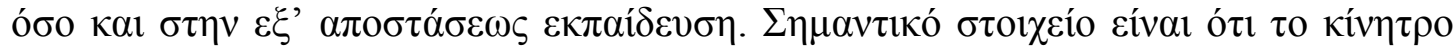

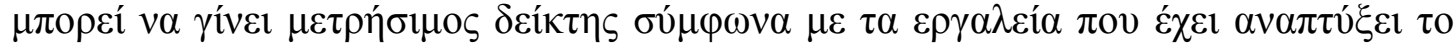

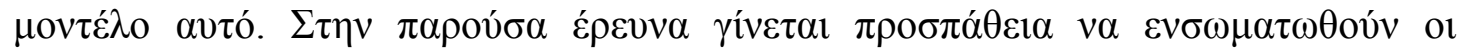

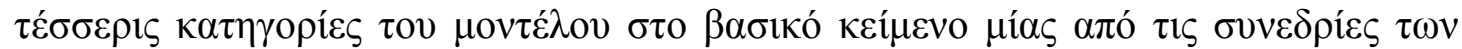

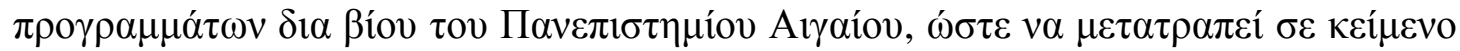

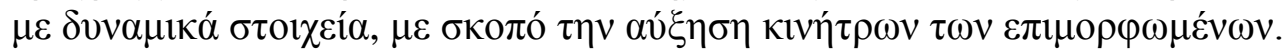

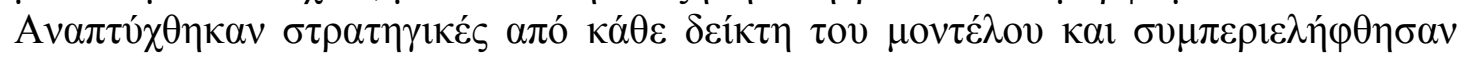

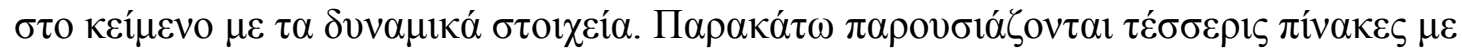

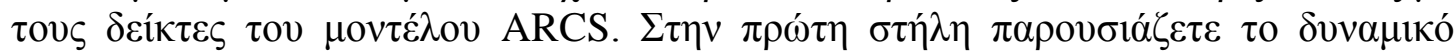

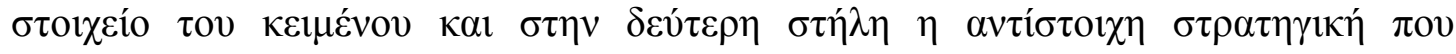

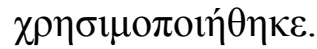




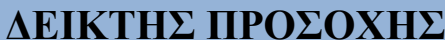

\begin{tabular}{|c|c|}
\hline 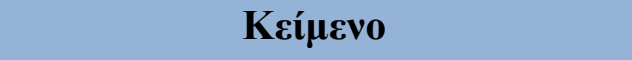 & 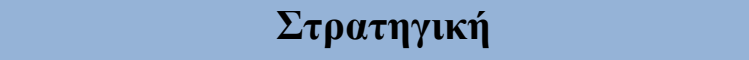 \\
\hline 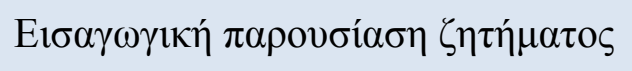 & $\Delta \mathrm{t} \varepsilon ́ \gamma \varepsilon \rho \sigma \eta \alpha \nu \tau i ́ \lambda \eta \psi \eta \varsigma(\mathrm{A} 1)$ \\
\hline 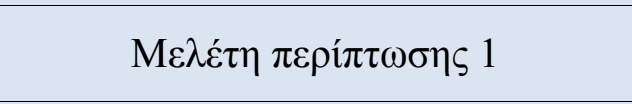 & 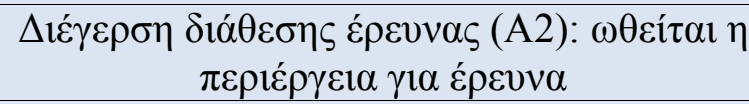 \\
\hline 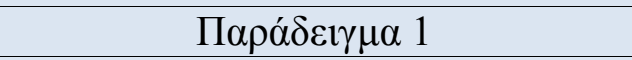 & 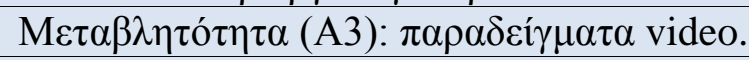 \\
\hline 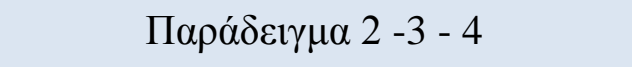 & 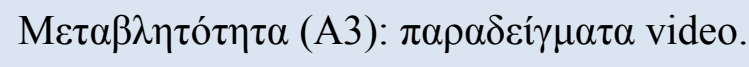 \\
\hline 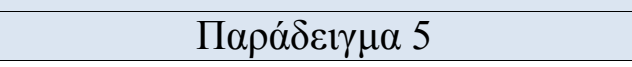 & 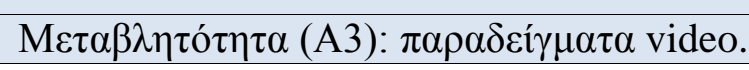 \\
\hline
\end{tabular}

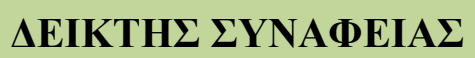

\begin{tabular}{|c|c|}
\hline Keí $\mu \varepsilon v o$ & $\Sigma \tau \rho \alpha \tau \eta \gamma \iota \kappa \eta ́$ \\
\hline 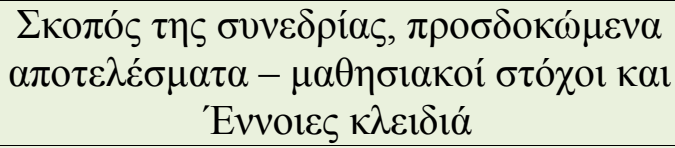 & 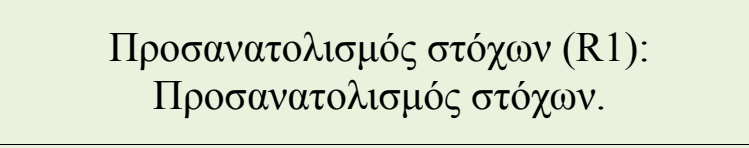 \\
\hline 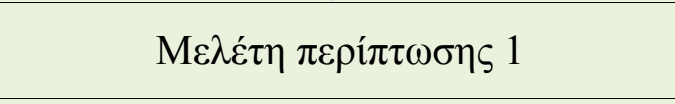 & 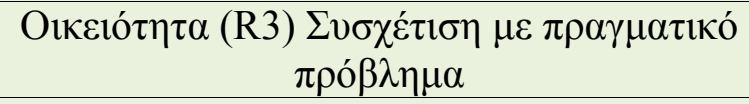 \\
\hline$\Delta \rho \alpha \sigma \tau \eta \rho \imath{ }^{\prime} \tau \tau \alpha 2$ & 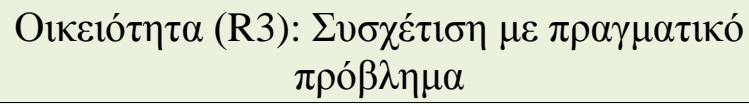 \\
\hline$\Delta \rho \alpha \sigma \tau \eta \rho \imath o ́ \tau \eta \tau \alpha 3$ & 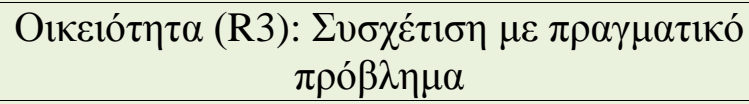 \\
\hline 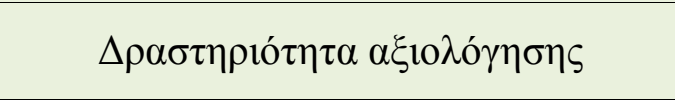 & 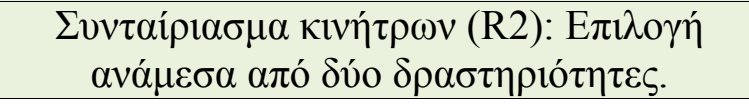 \\
\hline
\end{tabular}

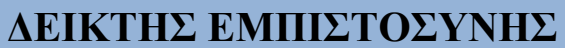

\begin{tabular}{|c|c|}
\hline Kєí $\mu \varepsilon v o$ & 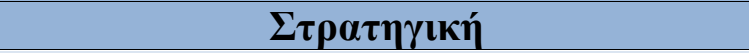 \\
\hline 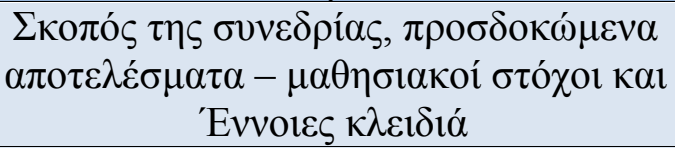 & 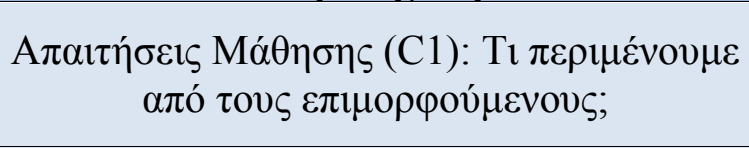 \\
\hline$\Delta \rho \alpha \sigma \tau \eta \rho ı ́(\eta \tau \alpha 1$ & 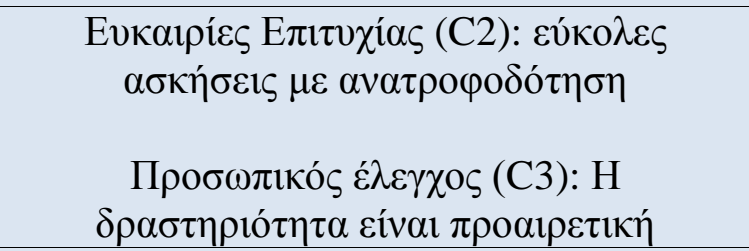 \\
\hline$\Delta \rho \alpha \sigma \tau \eta \rho ı ́(\eta \tau \alpha 2$ & 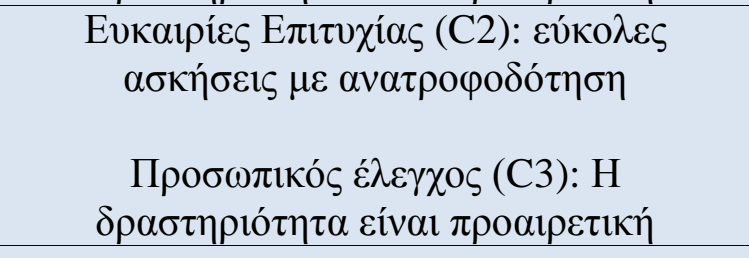 \\
\hline 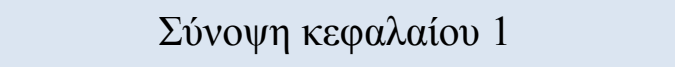 & 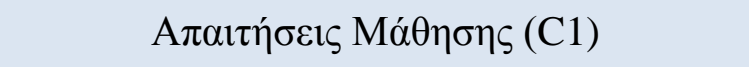 \\
\hline$\Delta \rho \alpha \sigma \tau \eta \rho i o ́ \tau \eta \tau \alpha 3$ & 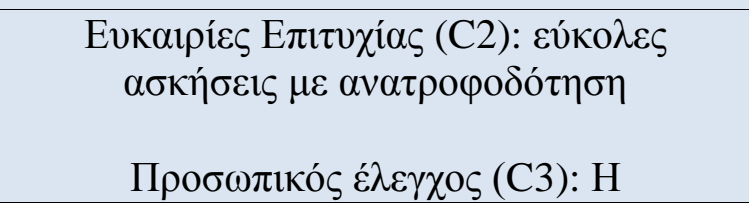 \\
\hline
\end{tabular}




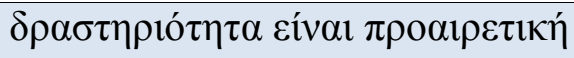

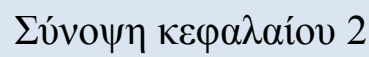

A $\pi \alpha \imath \eta \dot{\sigma \varepsilon \imath \varsigma ~ M \alpha ́ \theta \eta \sigma \eta \varsigma(C 1) ~}$

\begin{tabular}{|c|c|}
\hline \multicolumn{2}{|c|}{$\triangle$ EIKTH $\Sigma$ IKANOПOІH $\Sigma H \Sigma$} \\
\hline Keíleqvo & $\Sigma \tau \rho \alpha \tau \eta \gamma \iota \kappa \eta ́$ \\
\hline \multirow{3}{*}{$\Sigma u ́ v o \psi \eta ~ \kappa \varepsilon \varphi \alpha \lambda \alpha i ́ o v ~ 1$} & 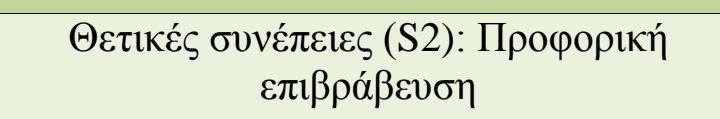 \\
\hline & \\
\hline & 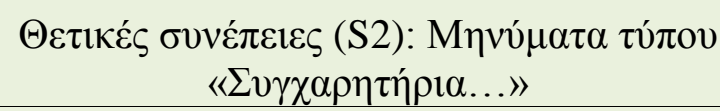 \\
\hline$\Delta \rho \alpha \sigma \tau \eta \rho i o ́ \tau \eta \tau \alpha 3$ & 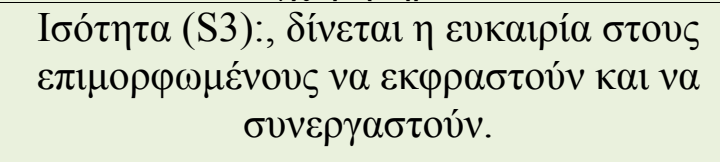 \\
\hline 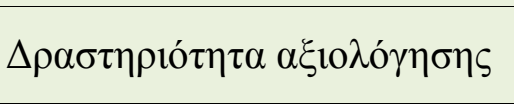 & 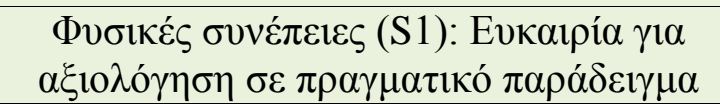 \\
\hline \multirow{3}{*}{ 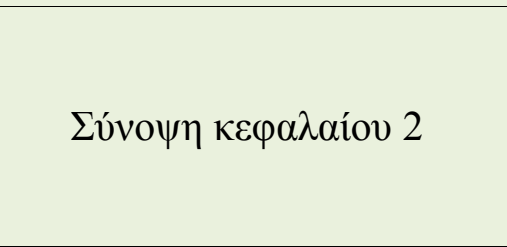 } & 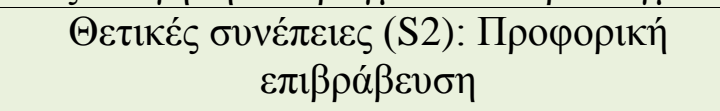 \\
\hline & \\
\hline & 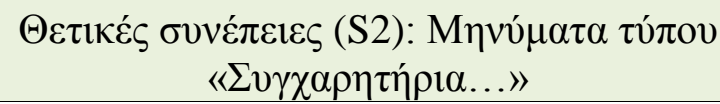 \\
\hline
\end{tabular}

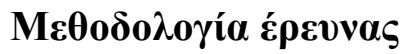

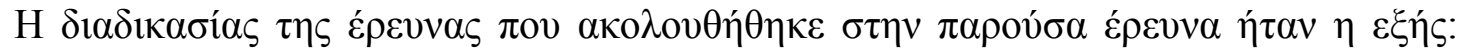

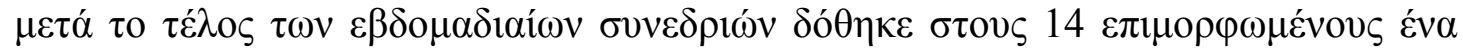

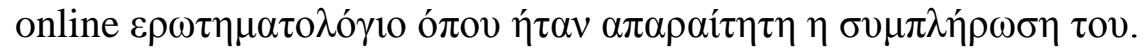

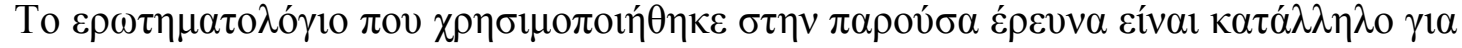

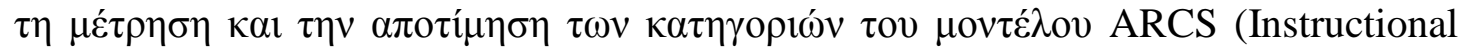

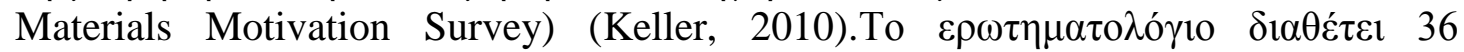

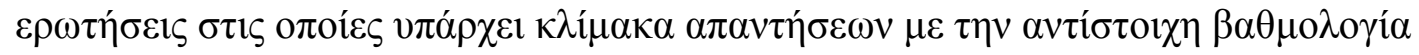

- $M \eta \alpha \lambda \eta \theta \dot{\varepsilon} \varsigma=1$

- $E \lambda \alpha \varphi \rho \omega ́ \varsigma \alpha \lambda \eta \theta \dot{\varepsilon} \varsigma=2$

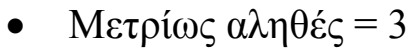

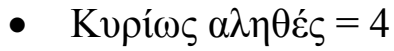

- Подv́ $\alpha \lambda \eta \theta \dot{\varepsilon} \varsigma=5$

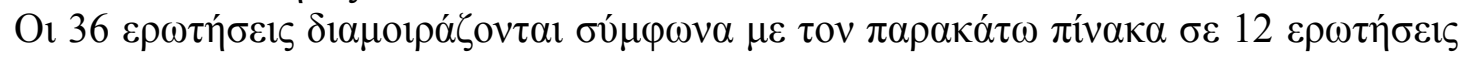

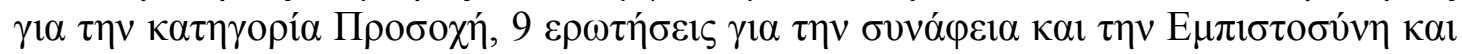

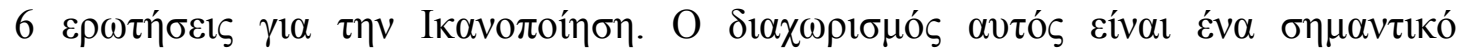

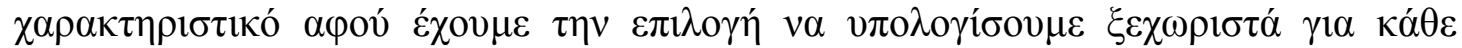

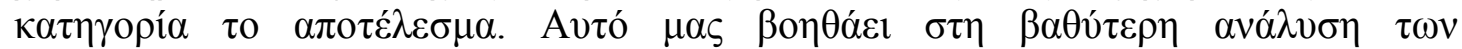

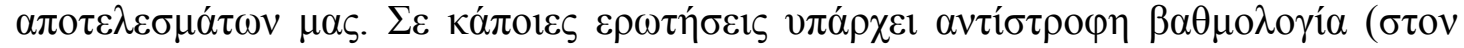

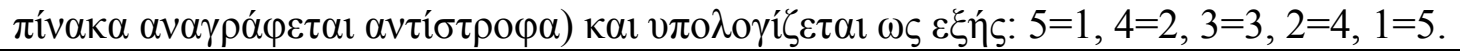

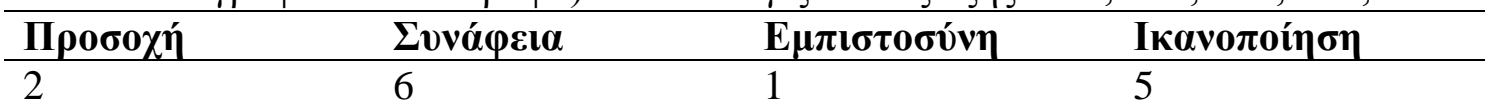




\begin{tabular}{|c|c|c|}
\hline 8 & 9 & $3(\alpha \nu \tau i ́ \sigma \tau \rho о \varphi \alpha)$ \\
\hline 11 & 10 & 4 \\
\hline $12(\alpha \nu \tau i ́ \sigma \tau \rho о \varphi \alpha)$ & 16 & $7(\alpha v \tau i ́ \sigma \tau \rho о \varphi \alpha)$ \\
\hline $15(\alpha \nu \tau i ́ \sigma \tau \rho о \varphi \alpha)$ & 18 & 13 \\
\hline 17 & 23 & $19(\alpha \nu \tau i ́ \sigma \tau \rho о \varphi \alpha)$ \\
\hline 20 & $26(\alpha \nu \tau i ́ \sigma \tau \rho о \varphi \alpha)$ & 25 \\
\hline 22 & 30 & $34(\alpha \nu \tau i ́ \sigma \tau \rho o \varphi \alpha)$ \\
\hline 24 & 33 & 35 \\
\hline \multicolumn{3}{|l|}{28} \\
\hline \multicolumn{3}{|l|}{$29(\alpha \nu \tau i ́ \sigma \tau \rho о \varphi \alpha)$} \\
\hline 31 & & \\
\hline
\end{tabular}

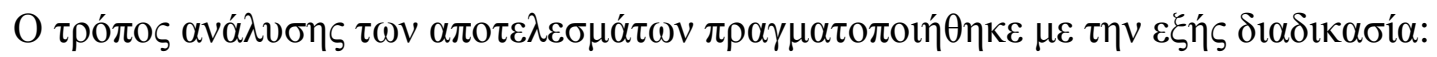

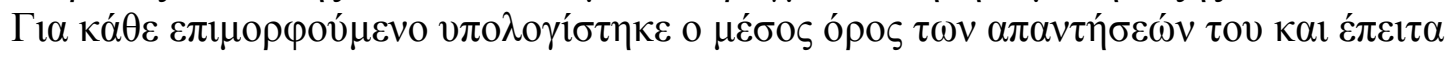

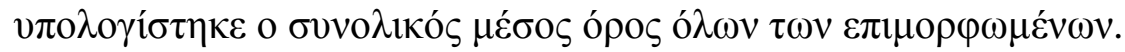

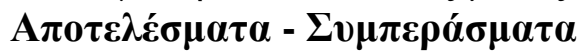

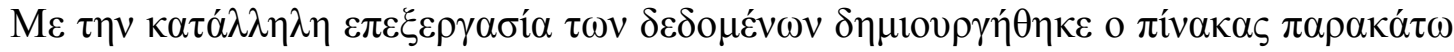

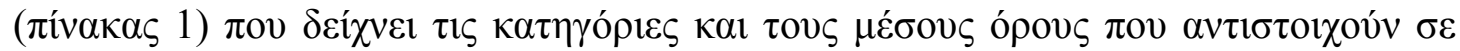

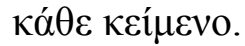

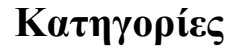

Пробохй

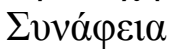

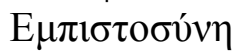

Ікаvолоі́ $\sigma \eta$
$\Delta v \operatorname{v\mu \iota \kappa ó~\kappa \varepsilon ́́\mu \varepsilon vo~}$

$\begin{array}{ll}4,1 & 3,4 \\ 3,9 & 3,2 \\ 4,1 & 2,9 \\ 4,2 & 2,9\end{array}$

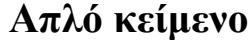

3,4

3,2

2,9

Пívokas 1

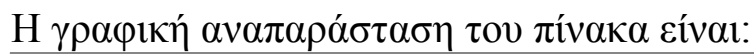

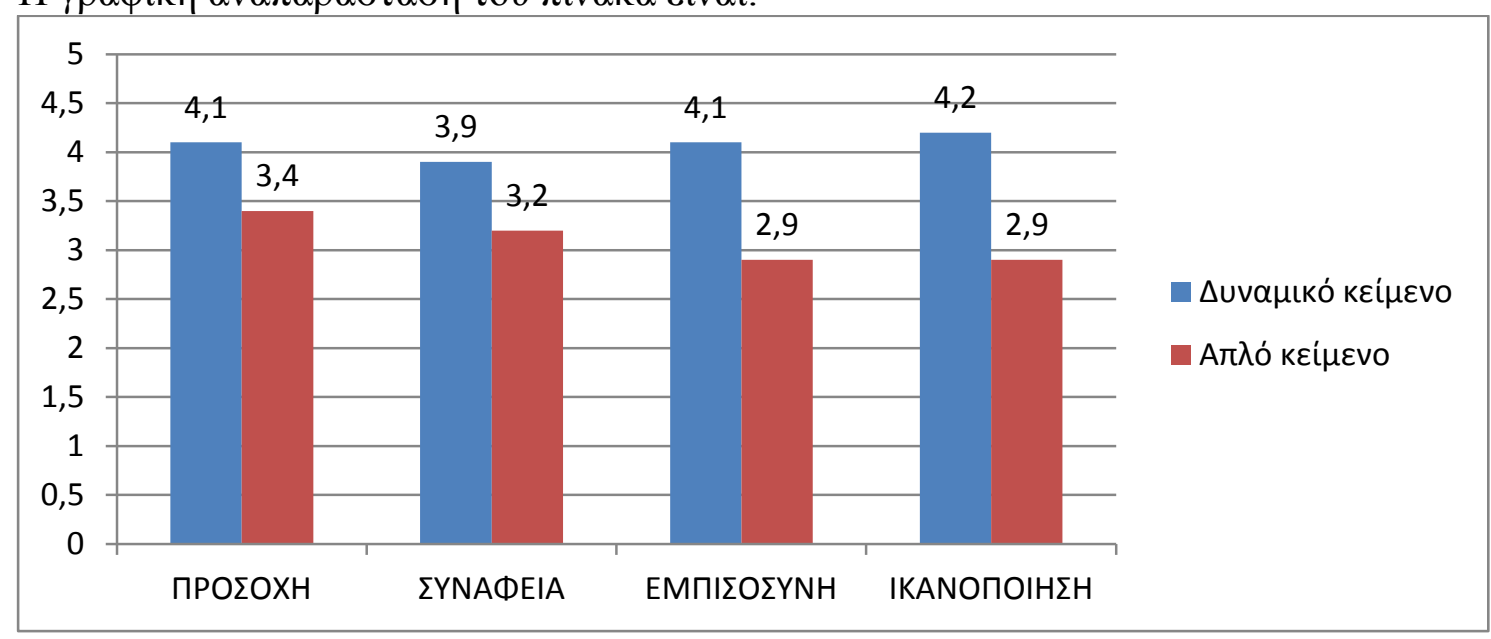

Гро́́

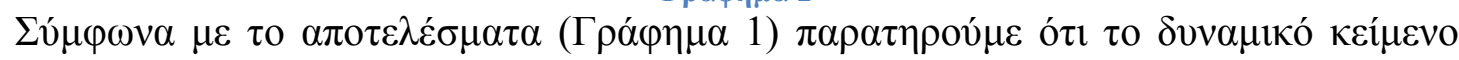

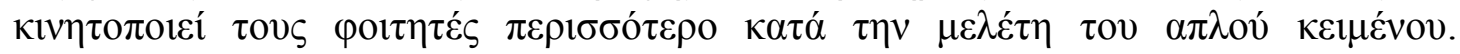

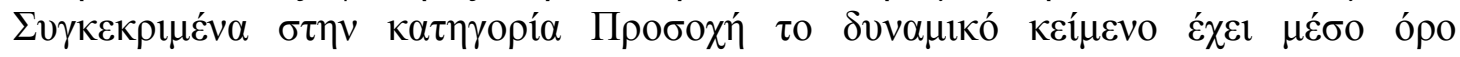

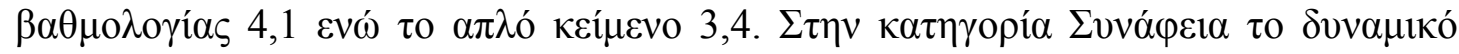

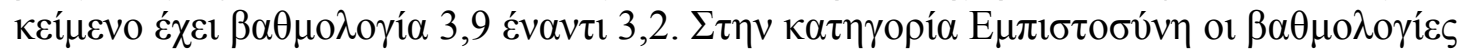

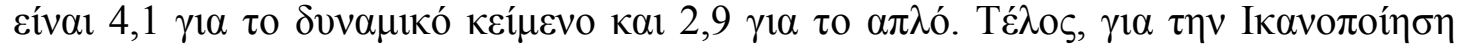

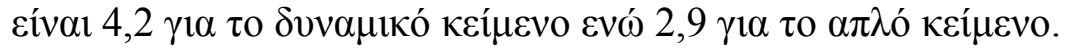

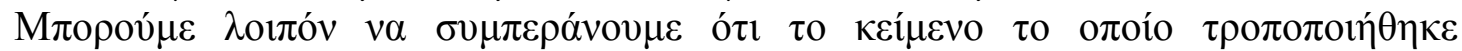

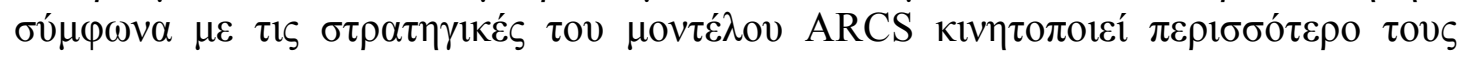




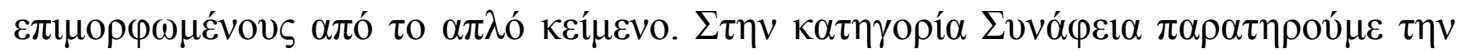

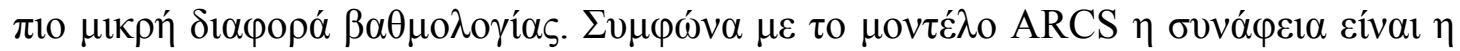

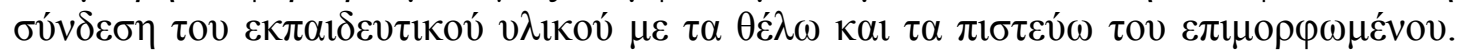

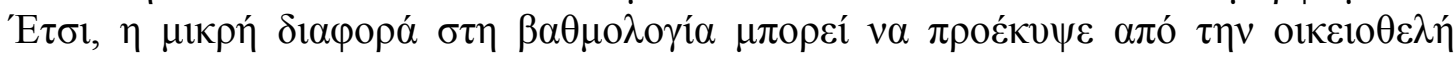

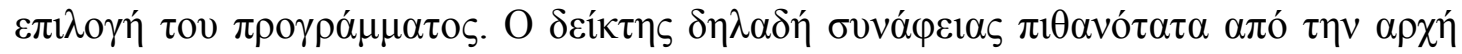

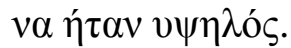

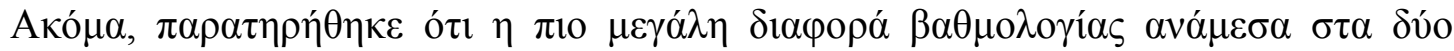

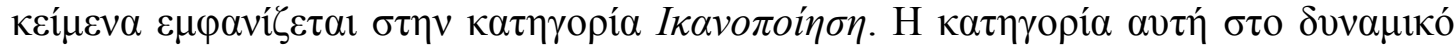

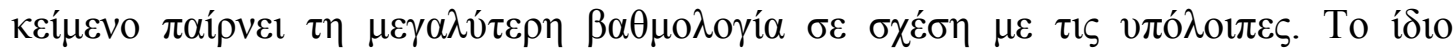

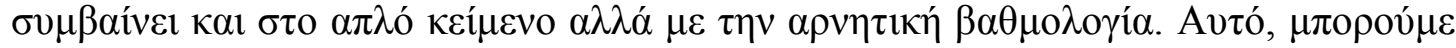

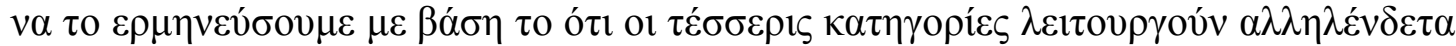

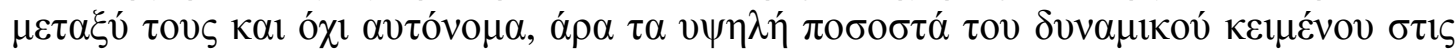

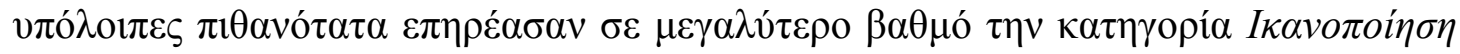

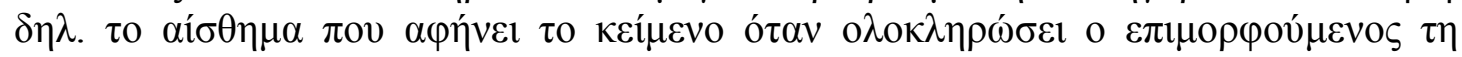

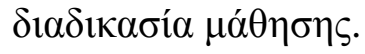

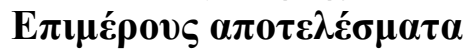

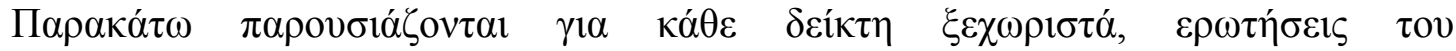

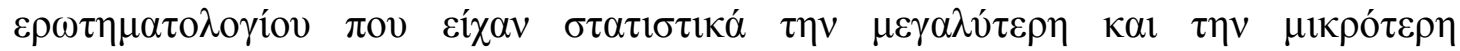

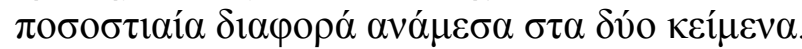

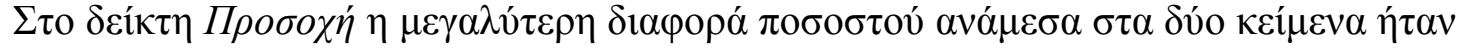

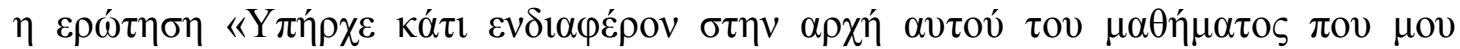

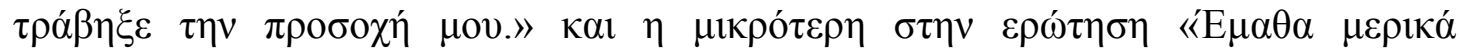

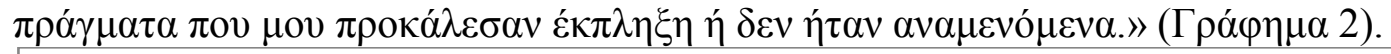

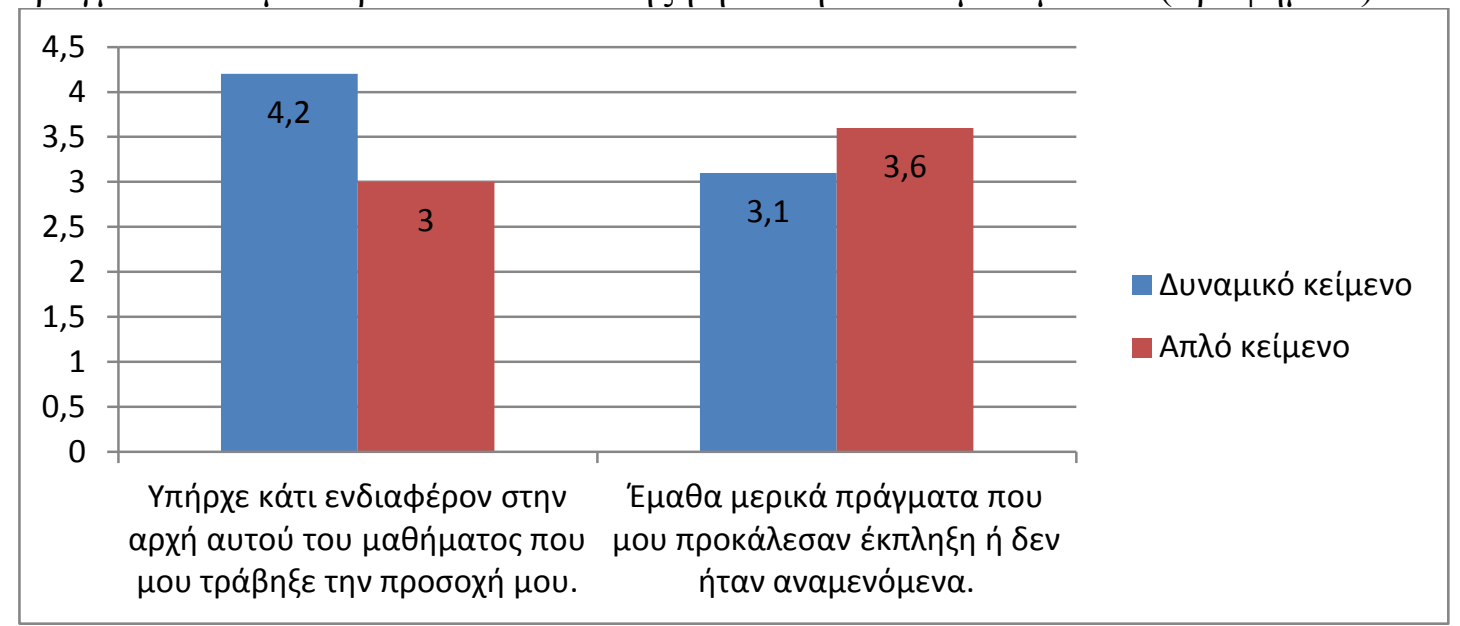

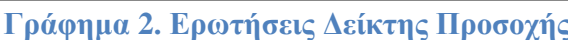

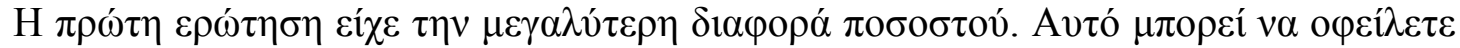

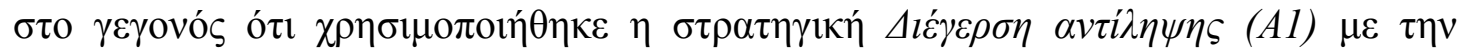

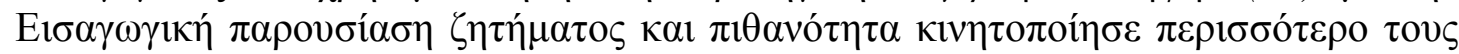
$\varepsilon \pi \mu о \rho \varphi \omega \mu \varepsilon ́ v o v \varsigma$.

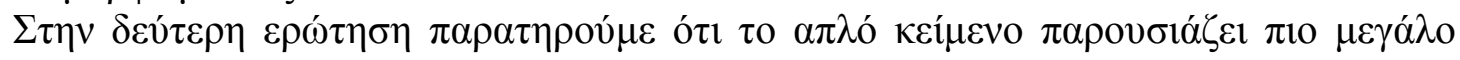

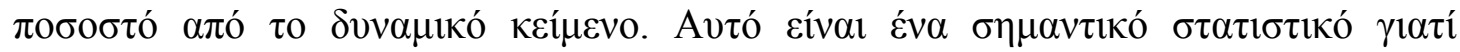

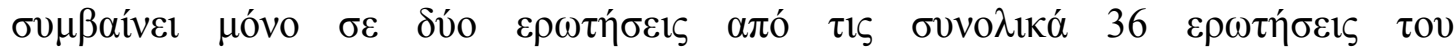

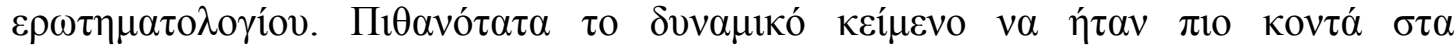

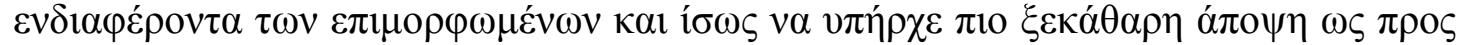

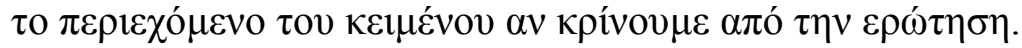

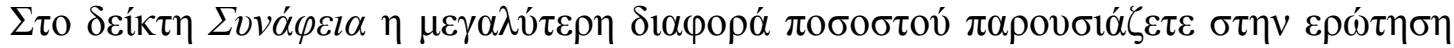

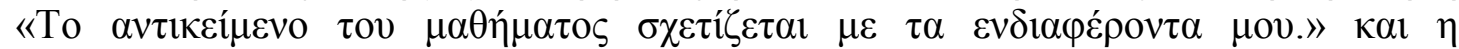




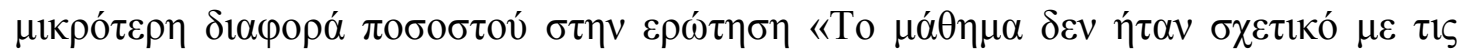

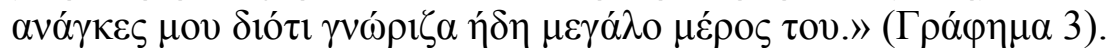

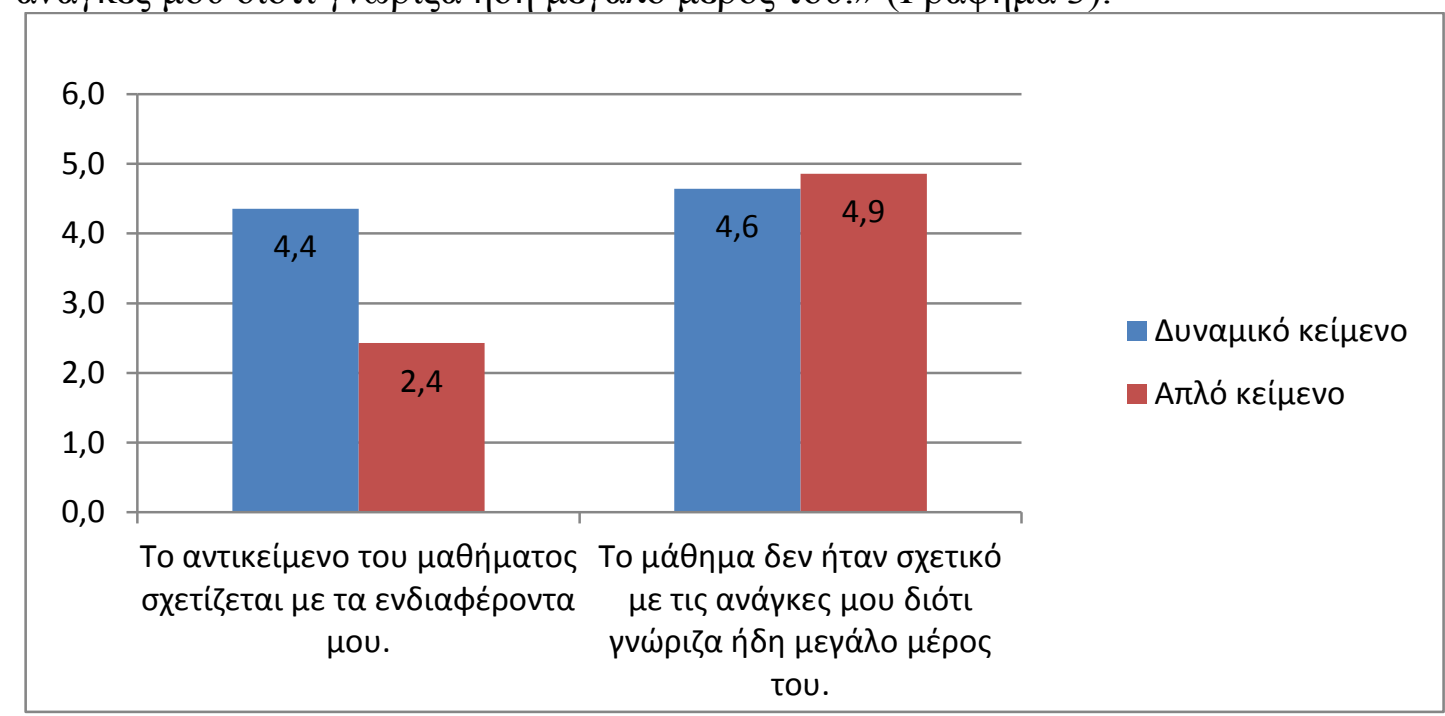

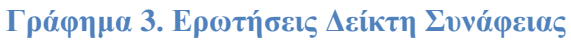

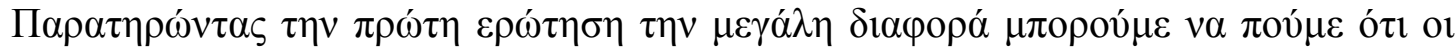

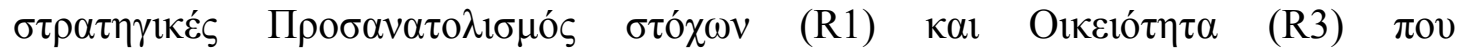

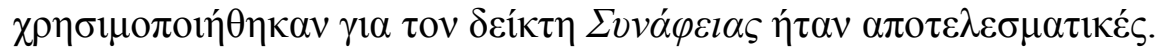

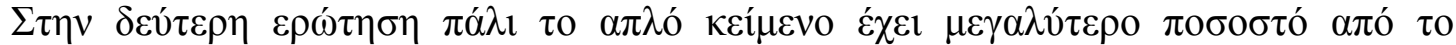

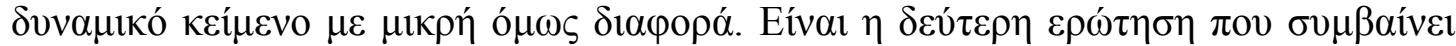

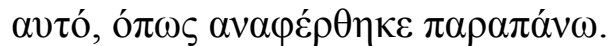

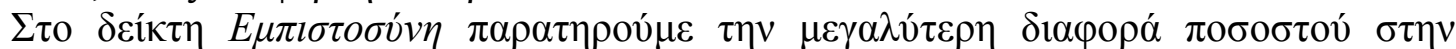

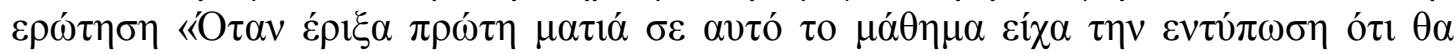

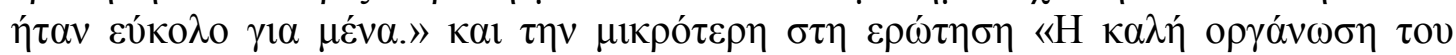

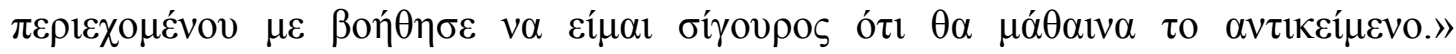

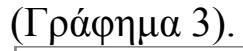

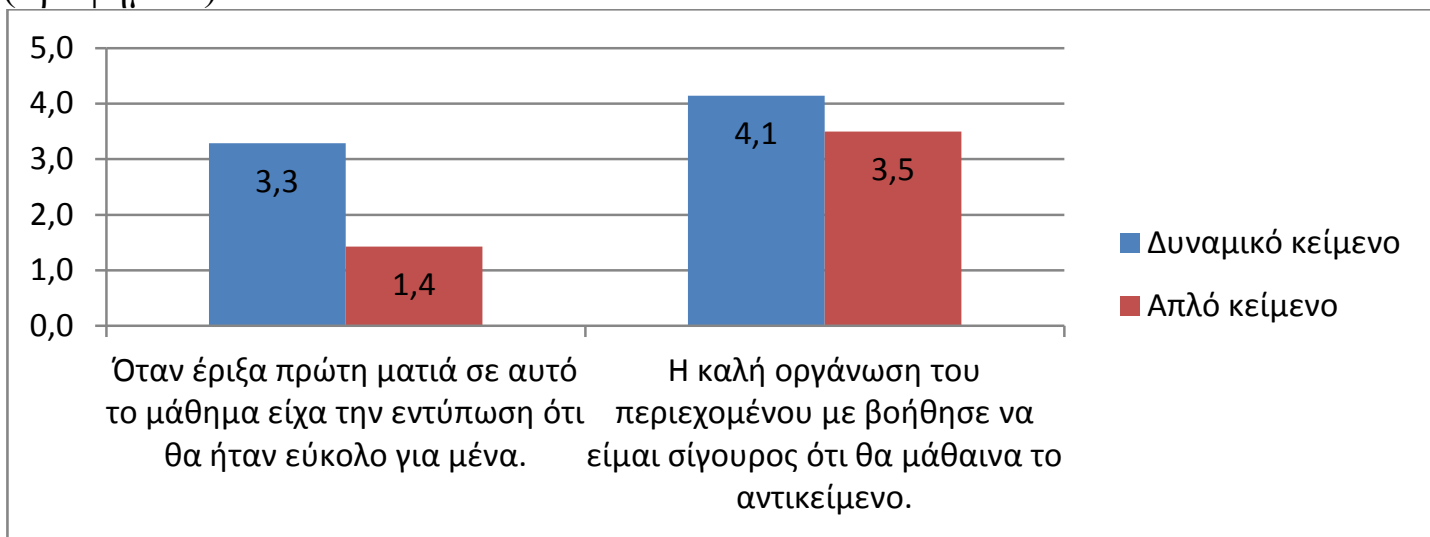

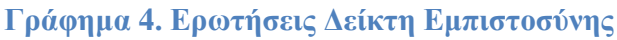

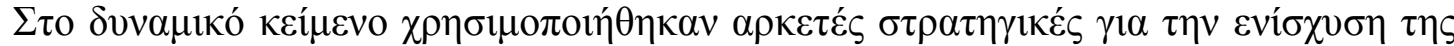

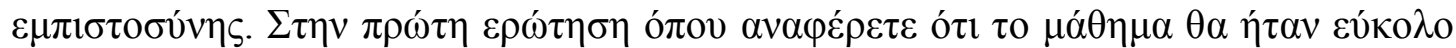

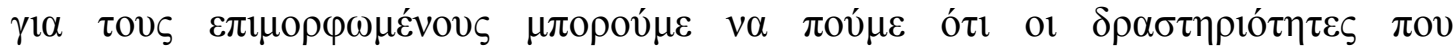

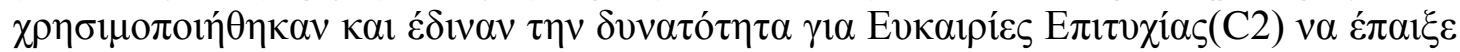

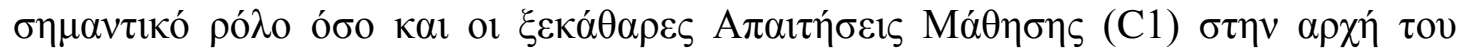

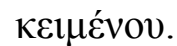

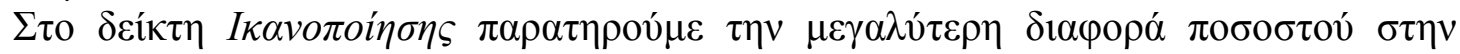

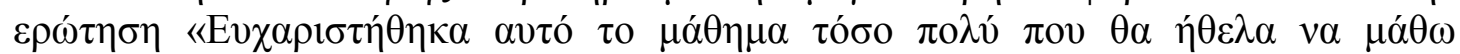

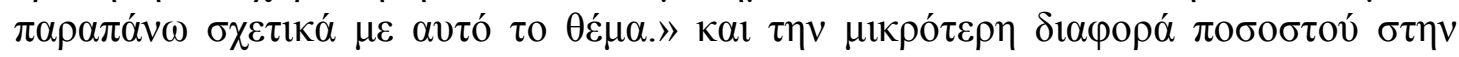




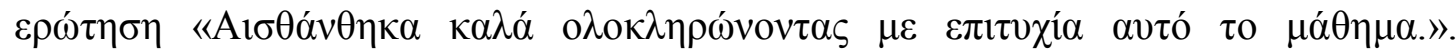
(Гра́

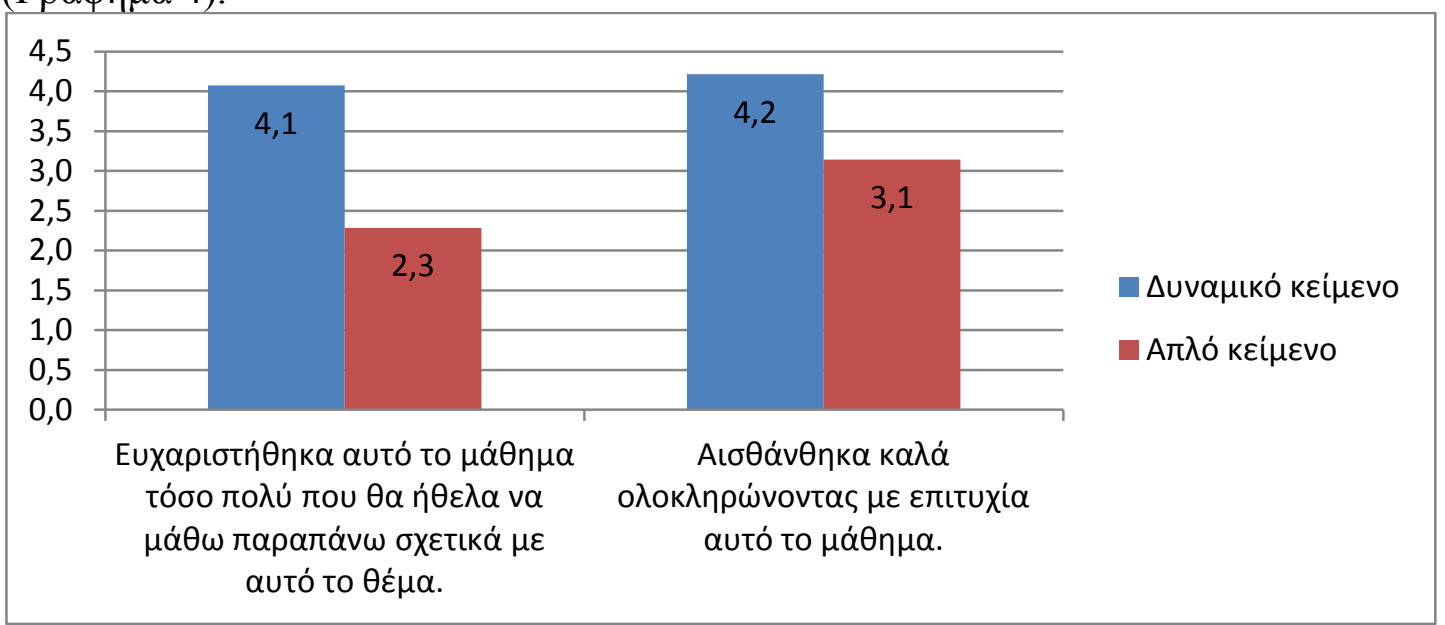

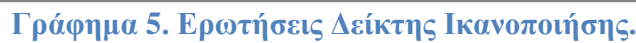

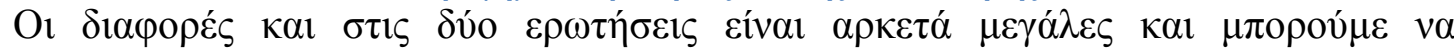

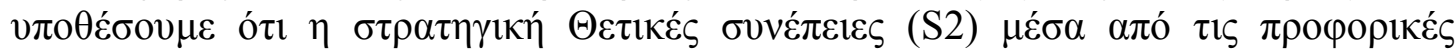

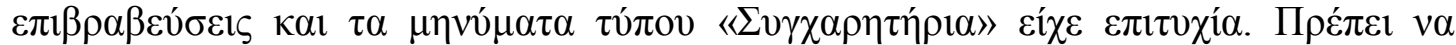

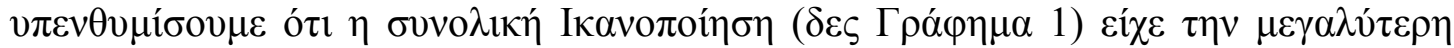

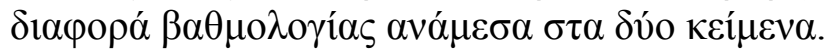

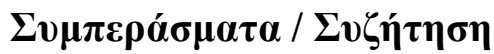

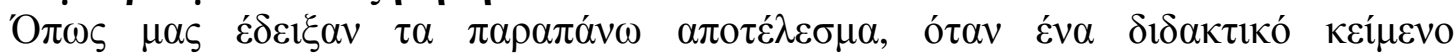

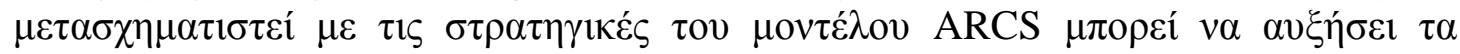

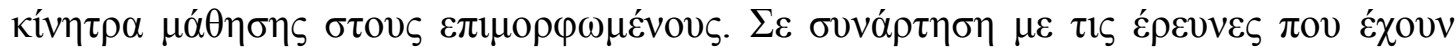

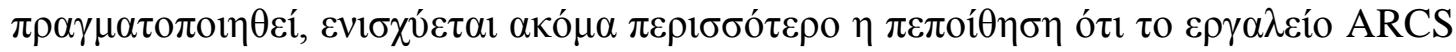

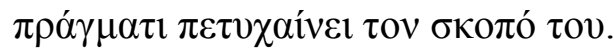

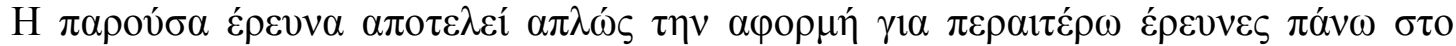

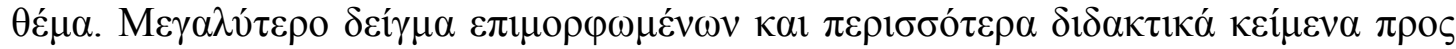

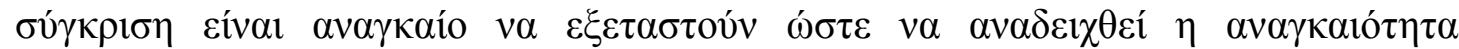

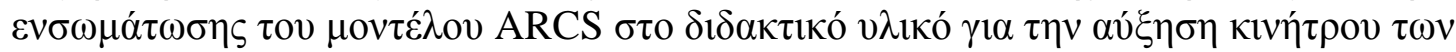
$\varepsilon \pi \mu о \rho \varphi \omega \mu \varepsilon ́ v \omega v$.

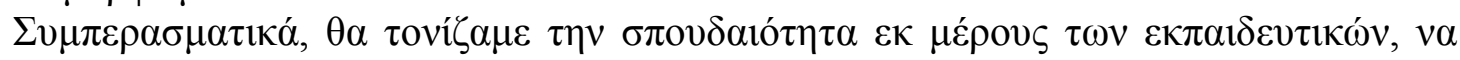

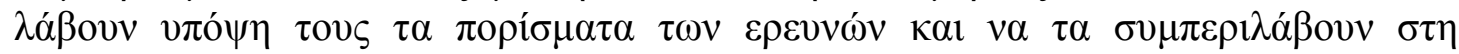

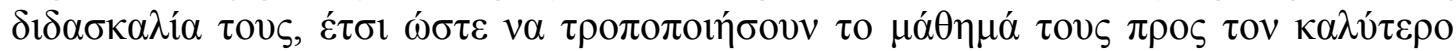

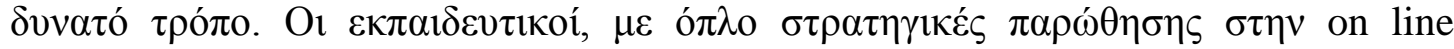

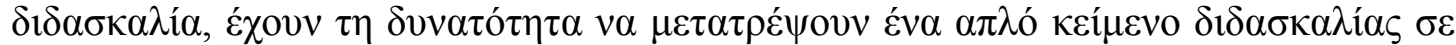

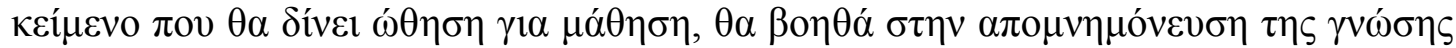

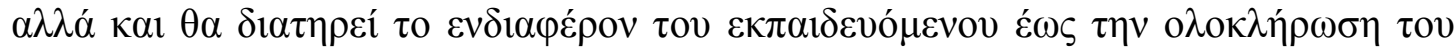
$\mu \alpha \theta \eta \dot{\mu} \mu \alpha \tau$ s.

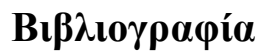

Astleitner, H. (2004). The effects of ARCS - strategies on self-regulated learning with instruxtional texts. Austria: University of Salzburg.

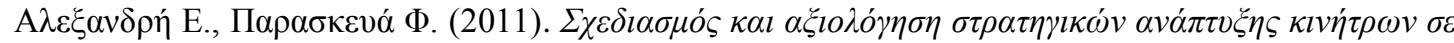

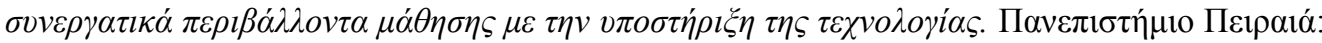

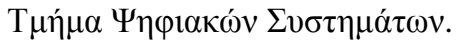


Balaban - Sali, J. (2008). Designing motivational learning systems in distance education, Turkish Online Journal of Distance Education. Anadolu University: TOJDE ISSN 1302-6488 Volume 9 Number 3 Article 13.

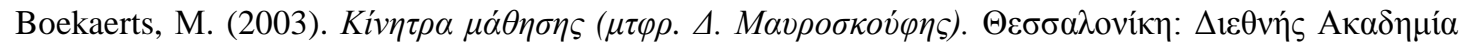

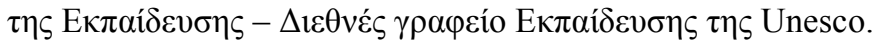

Huang D., Diefes-Dux H., P.K. Imbrie, Daku B., Kallimani J. (2002). Learning Motivation Evaluation for a Computer-based Instructional Tutorial Using ARCS Model of Motivational Design. Atlanta: $34^{\text {th }}$ ASEE/IEEE Frontiers in Education Conference.

Keller, J. (2010). Motivational Design for Learning and Perfomance The ARCS Model Aproach. New York: Springer Publication.

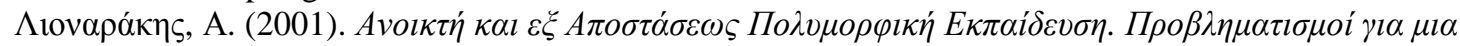

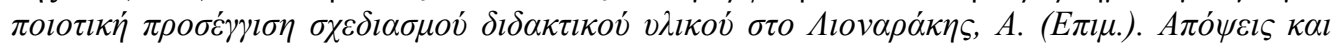

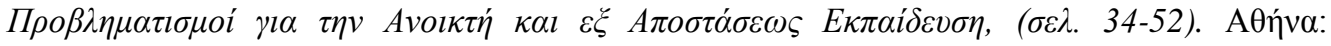
Пролонло́s.

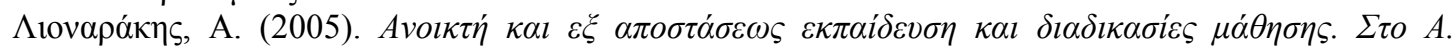

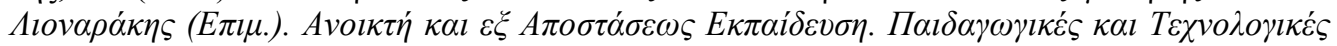

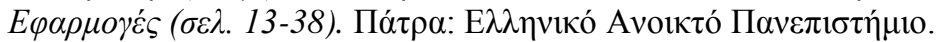

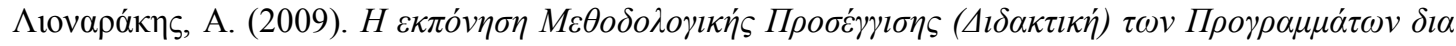

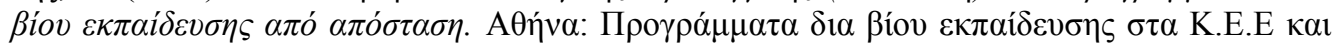
$\sigma \tau$ К K.E.E.EN.AП.

Poulsen A., Lam K., Cisneros S., Trust T. (2008). ARCS Model of Motivational Design. San Diego State University: EDTEC 544.

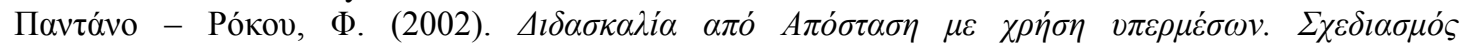

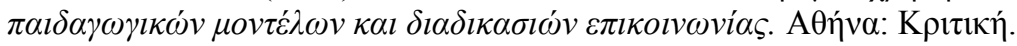

Rheinberg, F., Vollmeyer, R., \& Rollet, W. (2000). Motivation and action in self-regulated learning. In M. Boekaerts, P. R. Pintrich, \& M. Zeidner (Eds.), Handbook of self-regulation (pp. 503-529). San Diego, CA: Academic Press.

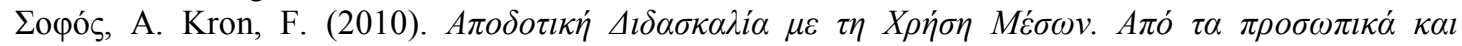

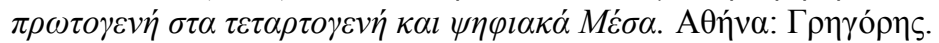

